\title{
A DIFFERENTIAL RICCATI EQUATION FOR THE ACTIVE CONTROL OF A PROBLEM IN STRUCTURAL ACOUSTICS
}

\author{
GEORGE AVALOS \\ INSTITUTE FOR MATHEMATICS AND ITS APPLICATIONS, UNIVERSITY OF MINNESOTA, \\ MINNEAPOLIS, MN 55455-0436. \\ IRENA LASIECKA \\ DEPARTMENT OF APPLIED MATHEMATICS, THORNTON HALL, UNIVERSITY OF VIRGINIA, \\ CHARLOTTESVILLE, VA 22903.
}

\begin{abstract}
In this paper, we provide results concerning the optimal feedback control of a system of partial differential equations which arises within the context of modelling a particular fluid/structure interaction system seen in structural acoustics, this application being the primary motivation for our work. This model onsists of two coupled PDE's exhibiting parabolic and hyperbolic characteristics respectively; the control action, in this case, is modelled by a highly unbounded operator. We rigorously justify a optimal control theory or this class of problems and characterize the optimal control through a suitable Riccati Equation. This is achieved, in part, by exploiting recent techniques in the area of optimization of analytic systems with unbounded inputs, along with a local microanalysis of the hyperbolic part of the dynamics, an analysis which will consider the propagation of singularities and optimal "trace" behavior of the solutions.
\end{abstract}

\section{INTRODUCTION}

1.1. Motivation. Let $\Omega$ be a bounded domain in $\mathbf{R}^{2}$ with Lipschitz boundary $\Gamma, \Gamma_{0}$ a Lipschitz segment of $\Gamma$ with endpoints $a$ and $b$, and $[s, T]$ some interval with $0 \leq s<T$. We consider the following problem (see [1],[2]) which requires that we find functions $z(t, x)$ and $v(t, x)$, corresponding to a fixed $u(t) \in U, U \equiv \mathbf{R}^{k}$, which solve the following system consisting of a coupled wave equation and elastic beam-like equation:

$$
\begin{aligned}
& z_{t t}=\Delta z \text { on } \Omega \times(s, T) ; \\
& \frac{\partial z}{\partial \nu}=\left\{\begin{array}{rr}
v_{t} & \text { on } \Gamma_{0} \times(s, T) \\
0 & \text { on } \Gamma \backslash \Gamma_{0} \times(s, T)
\end{array} ;\right. \\
& v_{t t}=-\Delta^{2} v-\Delta^{2} v_{t}-z_{t}+B u \text { on } \Gamma_{0} \times(s, T) ; \\
& v(a, t)=v(b, t)=\frac{\partial v(a, t)}{\partial x}=\frac{\partial v(b, t)}{\partial x}=0, \quad \forall t \in(s, T) ; \\
&\left\{\begin{array}{l}
z(s, x)=z_{0}, v(s, x)=v_{0} \\
z_{t}(s, x)=z_{1}, v_{t}(s, x)=v_{1}
\end{array}\right.
\end{aligned}
$$


here $B \in \mathcal{L}\left(U, H^{-\alpha}\left(\Gamma_{0}\right)\right)$, where $\alpha$ will be specified throughout to be

$$
\begin{aligned}
\alpha & \leq \frac{7}{4} \text { when } \Omega \text { is rectangular, } \\
\text { and } \alpha & \leq \frac{5}{3} \text { for } \Omega \text { an arbitrary smooth domain. }
\end{aligned}
$$

We will be looking for a triple $\left[z^{*}, v^{*}, u^{*}\right]^{T}$, which solves (1.1) and which minimizes a given performance index. Our main interest, however, is a feedback realization of the resulting optimal control via a solution of an appropriate Riccati equation.

In the special case when the $\Omega$ is rectangular and the operator $B$ is of the form $B=$ $\sum_{i=1}^{r} \alpha_{i} \delta^{\prime}\left(x_{i}\right)$, where $\delta^{\prime}\left(x_{i}\right)$ are derivatives of delta functions evaluated at " $x_{i}$ ", the model (1.1) was considered in [1]. The physical interpretation for this particular structure of the control operator is that its control action is realized by the strategic placement of piezoelectric ceramic patches on the (flexible) boundary $\Gamma_{0}$; a voltage is subsequently applied through these patches and the resulting bending moments can be interpreted as second derivatives of Heaviside functions. Note that the control operator is highly unbounded and defined only through distribution theory. This is, in fact, the main difficulty of the problem which was fully recognized in [26], wherein the analysis culminated in the existence and uniqueness of the solution $[z, v]^{T}$ of (1.1) (for fixed $u$ ) defined only in the sense of distributions. Our main goal here to show the well-posedness of (1.1) in this fully unbounded case within a given state space, i.e. $[z, v]^{T}$ may be taken as (continuous) functions; moreover, we wish to provide a rigorous theory of feedback control characterized by a solution $P$ to the Differential Riccati Equation, and in particular to reveal smoothing properties of the resulting gain operator $B^{*} P$ (despite the inherent unboundedness of $B^{*}$ ).

To accomplish our goal, we shall consider a more general version of this problem formulated within an abstract differential equation; the result will then derived for a broader class of problems whereby problem (1.1) will be deduced as a special case.

It should be noted that the key elements of our analysis rely on: (i) sharp new regularity properties of the traces of the hyperbolic part of the dynamics (eqn. (1.1a)); (ii) the theory of analytic semigroups and associated singular integrals which takes advantage of certain smoothing effects associated with the analytic part of dynamics (eqn. (1.1c)); and finally, (iii) recent results on the characterization of domains of fractional powers of the so-called elastic operators which in turn, allows for a crucial interplay between functional analytical and p.d.e. results.

1.2. Abstract Formulation. We wish to recast (1.1) into an appropriate functional analytical form for which we need the following facts and definitions:

(i) We set $A=-\Delta+I$, acting on $L^{2}(\Omega)$ with

$$
D(A)=\left\{z \in H^{2}(\Omega) \ni \frac{\partial u}{\partial \nu}=0 \text { on } \Gamma\right\} ;
$$

It is well known that $A$ is self-adjoint and positive definite, so fractional powers are well-defined. 
(ii) With $A$ as above, we also have from [11] that

$$
D\left(A^{\alpha}\right)=H^{2 \alpha}(\Omega), \quad 0 \leq \alpha \leq \frac{3}{4},
$$

(iii) We shall consequently use the identification of $D\left(A^{\frac{1}{2}}\right)$ with $H^{1}(\Omega)$ throughout. In the sequel we shall also consider $A$ as a continuous mapping of $D\left(A^{\frac{1}{2}}\right)$ into its topological dual $\left[D\left(A^{\frac{1}{2}}\right)\right]^{\prime}$, and we denote this new realization by the same symbol.

(iv) Using (iii) we can then define the (Neumann) map $N$ on $H^{s}(\Gamma)$ by setting $N g \equiv z \forall g \in H^{s}(\Gamma)$ and $s \geq-\frac{1}{2}$, where $z$ is the unique solution of the equation

$$
\langle A z, v\rangle_{\left[D\left(A^{\frac{1}{2}}\right)\right]^{\prime} \times D\left(A^{\frac{1}{2}}\right)}=\int_{\Omega} \nabla z \cdot \nabla v+\int_{\Omega} z v=\langle g, v\rangle_{H^{s}(\Gamma) \times\left[H^{s}(\Gamma)\right]^{\prime}}
$$

$\forall v \in D\left(A^{\frac{1}{2}}\right)$. By elliptic regularity (see [4]), we will then have that

$$
N \in \mathcal{L}\left(H^{s}(\Gamma), H^{s+\frac{3}{2}}(\Omega)\right) .
$$

(v) $\gamma_{0}: H^{1}(\Omega) \rightarrow H^{\frac{1}{2}}(\Gamma)$ will denote the usual Sobolev trace map, and $\gamma: H^{1}(\Omega) \rightarrow H^{\frac{1}{2}}\left(\Gamma_{0}\right)$ will be defined as the restriction of $H^{\frac{1}{2}}(\Gamma)$ (the range of $\gamma_{0}$ ) to $\Gamma_{0}$, i.e.

$$
\gamma(v)=\left.v\right|_{\Gamma_{0}}, \quad \forall v \in H^{1}(\Omega) .
$$

Thus we will have from (1.5) that $\forall g \in L^{2}\left(\Gamma_{0}\right)$ and $v \in D\left(A^{\frac{1}{2}}\right)$,

$$
\langle A N g, v\rangle_{\left[D\left(A^{\frac{1}{2}}\right)\right]^{\prime} \times D\left(A^{\frac{1}{2}}\right)}=\left(g,\left.v\right|_{\Gamma_{0}}\right)_{L^{2}\left(\Gamma_{0}\right)},
$$

i.e.,

$$
\gamma=N^{*} A \text { as elements in } \mathcal{L}\left(D\left(A^{\frac{1}{2}}\right), L^{2}\left(\Gamma_{0}\right)\right)
$$

(vi) We set

$$
\AA=\Delta^{2}, D(\AA)=H^{4}\left(\Gamma_{0}\right) \cap H_{0}^{2}\left(\Gamma_{0}\right) ;
$$

$\AA$ is also self-adjoint and positive definite on $L^{2}\left(\Gamma_{0}\right)$, so fractional powers are welldefined. By [11] we have

(vii)

$$
D\left(\AA^{\alpha}\right)=H_{0}^{4 \alpha}\left(\Gamma_{0}\right), \quad 0 \leq \alpha<\frac{5}{8},
$$

$$
\begin{aligned}
A_{1} & \equiv\left[\begin{array}{cc}
0 & I \\
-A+I & 0
\end{array}\right]: D\left(A_{1}\right) \rightarrow H_{1} \quad \text { with } \\
D\left(A_{1}\right) & =\left\{\left[z_{1}, z_{2}\right]^{T} \in D(A) \times D\left(A^{\frac{1}{2}}\right)\right\} \\
H_{1} & \equiv D\left(A^{\frac{1}{2}}\right) \times L_{2}(\Omega)=H^{1}(\Omega) \times L_{2}(\Omega) .
\end{aligned}
$$


(viii)

(ix)

$$
\begin{aligned}
A_{0} & \equiv\left[\begin{array}{cc}
0 & I \\
-\AA & -\AA
\end{array}\right]: D\left(A_{0}\right) \rightarrow H_{0} \quad \text { with } \\
D\left(A_{0}\right) & =\left\{\left[v_{1}, v_{2}\right]^{T} \in\left[D\left(\AA^{\frac{1}{2}}\right)\right]^{2} \ni v_{1}+v_{2} \in D(\AA)\right\} ; \\
H_{0} & \equiv D\left(\AA^{\frac{1}{2}}\right) \times L_{2}\left(\Gamma_{0}\right)=H_{0}^{2}\left(\Gamma_{0}\right) \times L_{2}\left(\Gamma_{0}\right) .
\end{aligned}
$$

$$
C=\left[\begin{array}{cc}
0 & 0 \\
0 & \gamma^{*}
\end{array}\right] \in \mathcal{L}\left(H_{0},\left[D\left(A^{\frac{1}{2}}\right)\right]^{\prime} \times\left[D\left(A^{\frac{1}{2}}\right)\right]^{\prime}\right)
$$

(note that $\gamma^{*}=A N$ ); by duality with respect to $H_{1} \times H_{0}$, we then have

$$
C^{*}=\left[\begin{array}{cc}
0 & 0 \\
0 & N^{*} A
\end{array}\right] \in \mathcal{L}\left(\left[D\left(A^{\frac{1}{2}}\right) \times D\left(A^{\frac{1}{2}}\right), H_{0}^{*}\right)\right) .
$$

$(\mathrm{x})$

$$
\begin{aligned}
\mathcal{A} \equiv & {\left[\begin{array}{cc}
A_{1} & C \\
-C^{*} & A_{0}
\end{array}\right], \mathcal{A}: D(\mathcal{A}) \mapsto H_{1} \times H_{0} \text { where } } \\
D(\mathcal{A})= & \left\{\left[z_{1}, z_{2}, v_{1}, v_{2}\right]^{T} \in\left[D\left(A^{\frac{1}{2}}\right)\right]^{2} \times\left[D\left(\AA^{\frac{1}{2}}\right)\right]^{2}\right. \text { such that } \\
& \left.-z_{1}+N v_{2} \in D(A) \text { and such that } v_{1}+v_{2} \in D(\AA)\right\} .
\end{aligned}
$$

(xi)

$$
\mathcal{B}=[0,0,0, B]^{T} \in \mathcal{L}\left(U, H_{1} \times D\left(\AA^{\frac{1}{2}}\right) \times H^{-\alpha}\left(\Gamma_{0}\right)\right) .
$$

From $(1.14 \mathrm{a}-\mathrm{c})$, we can proceed to show a fortiori that $(\lambda-\mathcal{A})^{-1} \mathcal{B} \in \mathcal{L}\left(U, H_{1} \times H_{0}\right)$ and consequently

$$
\mathcal{B} \in \mathcal{L}\left(U,\left[D\left(\mathcal{A}^{*}\right)\right]^{\prime}\right) .
$$

So setting $Y \equiv\left[z, z_{t}, v, v_{t}\right]^{T}$ and $Y_{0} \equiv\left[z_{0}, z_{1}, v_{0}, v_{1}\right]^{T}$, we can then use (1.10) and the operator definitions above to rewrite (1.1) as the abstract dynamical system

$$
\begin{aligned}
\frac{d Y}{d t} & =\mathcal{A} Y+\mathcal{B} u \text { in }\left[D\left(\mathcal{A}^{*}\right)\right]^{\prime}, \\
Y(s) & =Y_{0} \in H_{1} \times H_{0} .
\end{aligned}
$$

Henceforth our attention is drawn toward solving (1.15).

Along with the abstract equation (1.15), we associate a quadratic functional

$$
J_{s}(Y, u)=\frac{1}{2} \int_{s}^{T}\left[\|R Y(t)\|_{Z}^{2}+\|u(t)\|_{U}^{2}\right] d t
$$


where $Z$ is some Hilbert space and $R \in \mathcal{L}\left(H_{1} \times H_{0}, Z\right)$ (WLOG we can take $Z$ to be self-dual). The optimal control problem associated to (1.15) is then defined as: Find

$$
\begin{aligned}
& {\left[\vec{z}^{*}\left(\cdot, s ; Y_{0}\right), \vec{v}^{*}\left(\cdot, s ; Y_{0}\right), u^{*}\left(\cdot, s ; Y_{0}\right)\right]^{T}} \\
& \quad=\left[Y^{*}\left(\cdot, s ; Y_{0}\right), u^{*}\left(\cdot, s ; Y_{0}\right)\right]^{T} \in L^{2}\left(s, T ; H_{1} \times H_{0}\right) \times L^{2}(s, T ; U)
\end{aligned}
$$

which minimizes (1.16) over all $[\vec{z}, \vec{v}, u]^{T}=[Y, u]^{T}$ which solves (1.15), where the control operator $\mathcal{B}$ is linear, bounded: $U \rightarrow H_{1} \times D\left(\AA^{\frac{1}{2}}\right) \times\left[D\left(\AA^{\frac{\alpha}{4}}\right)\right]^{\prime}$, (from the characterization $(1.10))$.

1.3. Literature. Problems related to feedback control and Riccati equations for the case of unbounded control actions have received considerable attention in the past. In fact, control problems described by analytic semigroups with fully unbounded control operators $B$ have a most comprehensive treatment in the literature (see [9],[16]). Also, in the case of hyperbolic problems with a trace-type assumption imposed upon the control operator $B$, it has been shown that the Algebraic Riccati Equation is solvable with the gain operator usually unbounded albeit densely defined (see [7],[10],[24]). The hyperbolicity of the p.d.e. is critical in the analysis of the gains, the boundedness of which can be achieved under additional smoothing-type hypotheses imposed upon the observation $R$ (as in [7],[3] and references therein). Needless to say, our problem here does not fall into any of these categories; more importantly, the techniques developed in these cited works are not readily adaptable to the present situation. The reasons we cannot appeal to the earlier theory are threefold:

(i) Our problem consists of coupled hyperbolic/"parabolic-like" equations with an unbounded control operator; thus techniques developed specifically for parabolic or hyperbolic cases are no longer applicable.

(ii) The coupling between the two dynamics is represented by an unbounded (trace-type) operator. This is a source of major difficulties in the treatment.

(iii) The observation $R$ is not assumed here to be smoothing, and thus there is no benefit of an additional regularity resulting from optimization (unlike the other treatments noted above).

In view of the above, new techniques dealing with the novel facets of the problem need to be developed. We note that sharp regularity of hyperbolic traces (which was necessarily studied by the authors) plays an absolutely major role. In fact, our final result, asserting the solvability of the Riccati Equation with the implementation of a bounded gain operator, clearly indicates an interplay between the degree of unboundedness of $B$ which can be allowed in the problem and the geometry of the domain which dictates the regularity of the traces (see Theorem 1.2).

1.4. Statement of Main Results. With reference to the control system described by

(i) the abstract evolution equation (1.15) with $\mathcal{A}$ and $\mathcal{B}$ as given by (1.14);

(ii) the associated performance index (1.16);

the following results hold true with $\alpha$ as specified in (1.1f):

Theorem 1.1. For all prescribed initial data $Y_{0} \in H_{1} \times H_{0}$, there exists a unique optimal control and trajectory

$$
u^{*}\left(\cdot, s ; Y_{0}\right) \underset{5}{\in} L^{2}(s, T ; U)
$$




$$
Y^{*}\left(\cdot, s ; Y_{0}\right) \in C\left([s, T] ; H_{1} \times H_{0}\right) .
$$

Moreover, the following (additional) regularity holds true:

$$
\begin{aligned}
u^{*}\left(\cdot, s ; Y_{0}\right) & \in C([s, T] ; U) ; \\
Y^{*}\left(\cdot, s ; Y_{0}\right) & \in C\left(\left([s, T] ; H_{1} \times H_{0}\right) \cap L^{2}\left(s, T ; H_{1} \times\left[D\left(\AA^{\frac{1}{2}}\right)\right]^{2}\right)\right.
\end{aligned}
$$

with continuous dependence on the data $Y_{0}$ (see Remark 2.4).

\section{Theorem 1.2.}

(i) There exists a self-adjoint, positive semidefinite operator $P(t)$,

$$
P(\cdot) \in \mathcal{L}\left(H_{1} \times H_{0}, C\left([0, T] ; H_{1}^{*} \times H_{0}^{*}\right)\right)
$$

(see Proposition 3.2).

(ii) $\forall t, 0 \leq t \leq T, \mathcal{B}^{*} P(t) \in \mathcal{L}\left(H_{1} \times H_{0}, U\right)$ with norm estimate

$$
\left\|\mathcal{B}^{*} P(t)\right\|_{\mathcal{L}\left(H_{1}^{*} \times H_{0}^{*}, U\right)} \leq C_{T}(T-t)^{1-\theta}
$$

where $\theta=\frac{\alpha}{4}$ (see Proposition 3.1.3(i)).

(iii) The minimum of the functional $J_{s}$ defined in (1.16), corresponding to the minimizer $\left[Y^{*}\left(\cdot, s ; Y_{0}\right), u^{*}\left(\cdot, s ; Y_{0}\right)\right]^{T}$, is

$$
J_{s}\left(Y^{*}\left(\cdot, s ; Y_{0}\right), u^{*}\left(\cdot, s ; Y_{0}\right)\right)=\left\langle P(t) Y_{0}, Y_{0}\right\rangle,
$$

where $\langle\cdot, \cdot\rangle$ denotes the duality pairing with respect to the $H_{1} \times H_{0}$-topology. See Proposition 3.1.4(iii).

(iv) For each $Y_{0} \in H_{1} \times H_{0}$ the optimal control $u^{*}\left(\cdot, s ; Y_{0}\right)$ is given in feedback form by

$$
u^{*}\left(t ; s ; Y_{0}\right)=-\mathcal{B}^{*} P(t) Y^{*}\left(t ; s ; Y_{0}\right), \quad 0 \leq s \leq t \leq T
$$

(see Proposition 3.1.3(ii)).

(v) For $0<t<T, P(t)$ satisfies the following Differential Riccati Equation (DRE) $\forall Y_{0}, Y_{1} \in$ $D(\mathcal{A})$ :

$$
\begin{aligned}
\left\langle\dot{P}(t) Y_{0}, Y_{1}\right\rangle= & -\left\langle R^{*} R Y_{0}, Y_{1}\right\rangle-\left\langle P(t) \mathcal{A} Y_{0}, Y_{1}\right\rangle \\
& -\left\langle P(t) Y_{0}, \mathcal{A} Y_{1}\right\rangle+\left(\mathcal{B}^{*} P(t) Y_{0}, \mathcal{B}^{*} P(t) Y_{1}\right)_{U}
\end{aligned}
$$

(see Lemma 3.2.2(i)).

(vi) The solution is unique within the class of self-adjoint $\hat{P}(t) \in \mathcal{L}\left(H_{1} \times H_{0}\right)$ such that $\forall Y \in H_{1} \times H_{0}$,

$$
\mathcal{B}^{*} \hat{P}(t) Y \in L^{\infty}(0, T ; U)
$$

(see Lemma 3.2.2(ii)).

Remarks 1.1. (1) Notice that time regularity of the optimal control given by (1.17a) is not intrinsic to PDE optimization problems with an unbounded control operator $\mathcal{B}$-it is an independent regularity result. In fact, one typically does not have continuity of the optimal controls. In our particular case, this enhanced regularity is a result of a smoothing effect of the analytic part of dynamics which is propagated onto the entire system in some sense to be made clear presently. 
(2) The key result behind the derivation of the Riccati Equation, in the general case of an unbounded operator $\mathcal{B}$, is the regularity of the gain operator $\mathcal{B}^{\star} P(t)$ (which appears in the quadratic term of the equation). In our present situation, it is shown (see Theorem 1.2(ii)) that the gain operator $\mathcal{B}^{*} P(t)$ is bounded, despite the unboundedness of $\mathcal{B}$ (and consequently $\left.\mathcal{B}^{*}\right)$.

(3) It is well known that in a purely hyperbolic case (with unbounded $\mathcal{B}$ ), the gain operators are intrinsically unbounded. What explains the regularity result Theorem 1.2(ii), in our particular case, is again, some propagation of regularity from the analytic part of the dynamics. This is probably the most technical part of the proof which requires an in depth "microlocal" analysis of traces to hyperbolic operators.

Remark 1.2. We notice that the problem considered in [1] is a special case of our more general setup where $\Omega$ is a rectangle,

$$
B u=\sum_{i=1}^{k} \delta^{\prime}\left(x_{i}\right) u_{i}, \quad x_{i} \in \Gamma_{0}, u_{i} \in \mathbf{R}, \forall i .
$$

By the Sobolev Imbedding Theorem, $\delta^{\prime}\left(x_{i}\right) \in H^{-\frac{3}{2}-\epsilon}\left(\Gamma_{0}\right)$, so the value of $\alpha=\frac{3}{2}+\epsilon<\frac{5}{3}$, and hence this particular unbounded input fits within the framework of our problem.

\section{Analysis of Open Loop Dynamics}

In this section we shall prove several properties related to the well-posedness of the open loop control system. These will be critical for the study of feedback control.

By appealing to [23], we have the following result regarding the dynamics generated by $\mathcal{A}$, and the consequent wellposedness of (1.1) in a weak sense.

Theorem 2.1. The operator $\mathcal{A}$ given by (1.14a-b) with $A_{0}, A_{1}$, and $C$ described by (1.11)(1.13) generates a strongly continuous semigroup on the Hilbert space $H_{1} \times H_{0}$.

2.1. The Control $\rightarrow$ State Map. The main goal of this section is to establish the regularity of the control $\rightarrow$ state map. That is to say that we will consider the map $u \rightarrow Y$ defined via the dynamical system (1.15) as

$$
Y(t)=e^{\mathcal{A} t} Y_{0}+\int_{0}^{t} e^{\mathcal{A}(t-\tau)} \mathcal{B} u(\tau) d \tau
$$

(WLOG we take the initial time $s=0$ ). Since $\mathcal{A}$ generates a $C_{0}$ semigroup on $H_{1} \times H_{0}$ and $\mathcal{B}: U \rightarrow\left[\mathcal{D}\left(\mathcal{A}^{*}\right)\right]^{\prime}$, the map $L$ defined by

$$
L u(t)=\int_{0}^{t} e^{\mathcal{A}(t-\tau)} \mathcal{B} u(\tau) d \tau \in \mathcal{L}\left(L^{2}(0, T ; U), C\left([0, T] ;\left[D\left(\mathcal{A}^{*}\right)\right]^{\prime}\right) .\right.
$$

However, the above a priori regularity is not sufficient for the subsequent analysis. We need more information concerning the "smoothing" properties of $L$. In fact, the main goal of this section, is to prove that

Lemma 2.1. $L \in \mathcal{L}\left(L^{2}(0, T ; U), C\left([0, T] ; H_{1} \times H_{0}\right)\right)$.

Remark 2.0. The result of Lemma 2.1 should be contrasted with that of [24] which shows that the operator $L$ is defined as a mapping into a space of distributions. 
The proof of Lemma 2.1 follows through a sequence of propositions. A critical role is played by the following results:

Theorem 2.A (see [6],[7]). Let $A_{0}$ be defined as in (1.12); then

(i) $A_{0}$ generates a $C_{0}$-semigroup of contractions $\left\{e^{A_{0}(\cdot)}\right\}$ which is also analytic on $H_{0}$ and $\rho\left(A_{0}\right) \subseteq\{\lambda \ni$ Re $\lambda \geq 0\}$.

(ii) Moreover, for $0 \leq \eta \leq \frac{1}{2}$ we have the following characterization of the fractional powers of $\left(-A_{0}\right)^{\eta}$ :

$$
D\left(\left(-A_{0}\right)^{\eta}\right)=D\left(\AA^{\frac{1}{2}}\right) \times D\left(\AA^{\eta}\right)
$$

(iii) The map

$$
e^{A_{0}(\cdot)} \in \mathcal{L}\left(H_{0},\left(L^{2}\left(0, T ;\left[D\left(\AA^{\frac{1}{2}}\right)\right]^{2}\right) .\right.\right.
$$

Remark 2.1. Note that $\left\{e^{A_{0}(\cdot)}\right\}_{t>0}$ has a greater "smoothing" effect on intial data then the standard results given for analytic semigroups (see [12], p.295).

The next theorem pertains to the regularity of solutions of the Neumann problem with given Dirichlet boundary data (the so-called Neumann- Dirichlet map); this result will be used in the work ahead.

Theorem 2.B. Let $z$ be a weak solution to the following Neumann BVP:

$$
\begin{aligned}
z_{t t} & =\Delta z \text { in } \Omega \times(0, T), \\
\frac{\partial z}{\partial \nu} & =g \in L^{2}\left(0, T ; H^{4 \beta}(\Gamma)\right) ; \text { with } \zeta \leq 4 \beta<\frac{1}{2}, \\
z(0) & =z_{t}(0)=0
\end{aligned}
$$

(where $\zeta=\frac{1}{4}$ if $\Omega$ is rectangular, and $\zeta=\frac{1}{3}$ in the general case of a smooth boundary $\Gamma$ ). Then we have that

$$
\left[z, z_{t},\left.z_{t}\right|_{\Gamma}\right]^{T} \in C\left([0, T] ; H^{1}(\Omega)\right) \times C\left([0, T] ; L^{2}(\Omega)\right) \times L^{2}\left(0, T ; H^{-4 \beta}(\Gamma)\right),
$$

with continuous dependence on the datum g; viz. $\forall t \in[0, T] \exists C$ (independent of $t$ ), such that

$$
\|z(t)\|_{H^{1}(\Omega)}^{2}+\left\|z_{t}(t)\right\|_{L^{2}(\Omega)}^{2}+\int_{0}^{T}\left\|z_{t}(s)\right\|_{H^{-4 \beta}(\Gamma)}^{2} d s \leq C\|g\|_{L^{2}\left(0, T ; H^{4 \beta}(\Gamma)\right)}^{2} .
$$

Remark 2.2. Theorem 2.B, in the case of rectangular domains was proved in [1]. In the case of "smooth" boundaries, the result of Theorem 2.B follows from techniques used in [15].

Remark 2.3. Notice that the result of Theorem 2.B states "sharp" regularity results for the Neumann-Dirichlet map which do not follow from standard PDE theory. In fact, the standard estimates (see [17]) require that $g \in H^{\frac{1}{2}}, \frac{1}{2}((0, T) \times \Gamma)$ in order to obtain only that $z \in C\left([0, T] ; H^{1}(\Omega)\right)$. Thus, our result improves the regularity by a $\frac{1}{2}-4 \beta$ derivative in space and a $\frac{1}{2}$ derivative in time. More recent and refined PDE estimates such as in [19] with initial 
datum $g \in L^{2}\left(0, T ; H^{\frac{1}{2}}(\Gamma)\right)$ produce $z \in C\left([0, T] ; H^{1}(\Omega)\right)$ and $z_{t} \in C\left([0, T] ; L^{2}(\Omega)\right)$. Our result in [1] still betters that of Myatake's by a $\frac{1}{2}-4 \beta$ space derivative (to obtain the same state regularity and, more importantly, by allowing for the "trace" $\left.z_{t}\right|_{\Gamma}$ to be defined in an appropriate negative Sobolev space. These above improvements for the Neumann-Dirichlet map are indispensable for the following analysis.

As $\mathcal{B} \in \mathcal{L}\left(U,\left[D\left(A^{*}\right)\right]^{\prime}\right)$, then $e^{\mathcal{A t}} \mathcal{B}$ is well defined as an element of $\left[D\left(\mathcal{A}^{*}\right)\right]^{\prime}$. Our main task now is to show that for $t>0, e^{\mathcal{A} t} \mathcal{B}$ may actually be taken as an element of $\mathcal{L}\left(U, H_{1} \times H_{0}\right)$.

\section{Proposition 2.2.1.}

(i) Let $\eta$ satisfy $\beta \leq \eta \leq \frac{1}{2}$, where $\beta$ is in the range $\zeta<\beta \leq \frac{1}{8}$ (where $\zeta=\frac{1}{16}$ if $\Omega$ is rectangular, and $\zeta=\frac{1}{12}$ if $\Gamma$ is a smooth arbitrary boundary), and define the map $K_{0}$ by having for every $\vec{v} \in L^{2}\left(0, T ; D\left(\left(-A_{0}\right)^{\eta}\right)\right.$,

$$
K_{0} \vec{v}(t)=\int_{0}^{t} e^{A_{0}(t-s)} C^{*} \int_{0}^{s} e^{A_{1}(s-\tau)} C v(\tau) d \tau d s
$$

then $K_{0} \in \mathcal{L}\left(L^{2}\left(0, T ; D\left(\left(-A_{0}\right)^{\eta}\right)\right)\right.$.

(ii) $I-K_{0}$ is boundedly invertible on $L^{2}\left(0, T ; D\left(\left(-A_{0}\right)^{\eta}\right)\right)$.

Proof of (i). From Theorem 2.A(i) above, we have that

$$
D\left(\left(-A_{0}\right)^{\beta}\right)=D\left(\AA^{\frac{1}{2}}\right) \times D\left(\AA^{\beta}\right),
$$

and from (1.10),

$$
D\left(\AA^{\eta}\right) \subseteq D\left(\AA^{\beta}\right)=H^{4 \beta}\left(\Gamma_{0}\right) ;
$$

we then note that for arbitrary $\vec{v}=\left[v, v_{t}\right]^{T} \in L^{2}\left(0, T ; D\left(\left(-A_{0}\right)^{\beta}\right)\right)$ the term

$$
\left[\begin{array}{c}
z(\cdot) \\
z_{t}(\cdot)
\end{array}\right] \equiv \int_{0}^{(\cdot)} e^{A_{1}(\cdot-\tau)}\left[\begin{array}{c}
0 \\
A N v_{t}(\tau)
\end{array}\right] d \tau=\int_{0}^{(\cdot)} e^{A_{1}(\cdot-\tau)} C \vec{v}(\tau) d \tau
$$

is a fortiori (see [15]) the unique (weak) solution of the following BVP:

$$
\begin{aligned}
z_{t t} & =\Delta z \\
\frac{\partial z}{\partial \nu} & =\left\{\begin{array}{rr}
v_{t} & \text { on } \Gamma_{0} \times(0, T) \\
0 & \text { on } \Gamma \backslash \Gamma_{0} \times(0, T) ;
\end{array} ;\right. \\
z(0) & =z_{t}(0)=0 .
\end{aligned}
$$

From the given regularity of $v_{t}$ we consequently deduce from Theorems 2.A(i), 2.B and the characterization $(2.7 \mathrm{~b})$ that the map

$$
\vec{z}(\cdot) \rightarrow C^{*} \int_{0}^{(\cdot)} e^{A_{1}(\cdot-\tau)} C \vec{v}(\tau) d \tau=\left(\begin{array}{c}
0 \\
z_{t_{\Gamma_{0}}}
\end{array}\right)
$$

is an element of

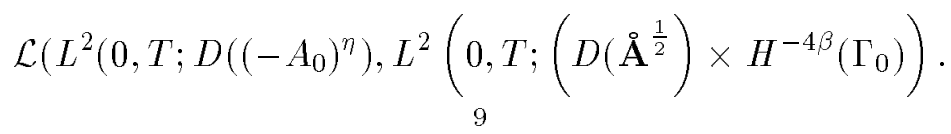


Now considering the inner integral of (2.5), if we define $j_{0}$ as the inclusion map from $H^{-4 \beta}\left(\Gamma_{0}\right)$ into $H^{-2}\left(\Gamma_{0}\right)=\left[D\left(\AA^{\frac{1}{2}}\right)\right]^{\prime}$ and set

$$
\mathcal{J}_{\frac{1}{2}}=\left[\begin{array}{cc}
0 & 0 \\
0 & j_{0}
\end{array}\right]
$$

then $\forall \vec{v}=\left[v, v_{t}\right]^{T} \in L^{2}\left(0, T ;\left(D\left(\AA^{\frac{1}{2}}\right)\right) \times H^{-4 \beta}\left(\Gamma_{0}\right)\right)$, we will have by the explicit representation of $\left(-A_{0}\right)^{\frac{1}{2}}$ given in $[13$, p.62] that

$$
\left(-A_{0}\right)^{-\frac{1}{2}} \mathcal{J}_{\frac{1}{2}} \vec{v}=\left[\begin{array}{l}
\AA^{-\frac{3}{4}}\left(2 I+\AA^{\frac{1}{2}}\right)^{-\frac{1}{2}} j_{0} v_{t} \\
\AA^{-\frac{1}{4}}\left(2 I+\AA^{\frac{1}{2}}\right)^{-\frac{1}{2}} j_{0} v_{t}
\end{array}\right] ;
$$

As $\AA$ is self-adjoint we then deduce that

$$
\left(-A_{0}\right)^{-\frac{1}{2}} \mathcal{J}_{\frac{1}{2}} \in \mathcal{L}\left(\left(D\left(\AA^{\frac{1}{2}}\right) \times H^{-4 \beta}, H_{0}\right) .\right.
$$

Consequently, we can make use of the well-developed theory for analytic semigroups (see [13]) (after agreeing to identify $j_{0}$ as simply the identity) to establish that the map

$$
\begin{aligned}
& \vec{v}(\cdot) \rightarrow \int_{0}^{(\cdot)} e^{A_{0}(\cdot-x)} \vec{v}(\tau) d \tau \\
& \quad \in \mathcal{L}\left(L^{2}\left(0, T ;\{0\} \times H^{-4 \beta}\left(\Gamma_{0}\right)\right)\right), L^{2}\left(0, T ; D\left(\left(-A_{0}\right)^{\frac{1}{2}}\right)\right) .
\end{aligned}
$$

As $D\left(\left(-A_{0}\right)^{\frac{1}{2}}\right) \subseteq D\left(\left(-A_{0}\right)^{\eta}\right)$, we have upon combining (2.10) and (2.13) that

$$
K_{0} \in \mathcal{L}\left(L^{2}\left(0, T ; D\left(\left(-A_{0}\right)^{\eta}\right)\right),\right.
$$

and (i) is proved.

(ii): We have further that the operator norm of $K_{0}$ is bounded by a constant dependent upon time. To wit, $\forall T_{0}, 0<T_{0} \leq T$, and $\vec{v} \in L^{2}\left(0, T_{0} ; D\left(\left(-A_{0}\right)^{\eta}\right)\right.$,

$$
\begin{aligned}
& \left\|\left(K_{0} \vec{v}\right)\right\|_{L^{2}\left(0, T_{0} ; D\left(\left(-A_{0}\right)^{\eta}\right)\right)} \\
& \quad \leq C_{0}\left\|K_{0} \vec{v}\right\|_{L^{2}\left(0, T ; D\left(\left(-A_{0}\right)^{\frac{1}{2}}\right)\right)} \\
& \quad \leq C_{T_{0}}\left\|C^{*} \int_{0}^{(\cdot)} e^{A_{1}(\cdot-\tau)} C \vec{v}(\tau) d \tau\right\|_{L^{2}\left(0, T_{0} ; H^{-4 \beta}\left(\Gamma_{0}\right)\right)}
\end{aligned}
$$

(by the norm estimate given in $[14, \mathrm{p} .455]$ and Theorem 2.B)

$$
\leq C_{T_{0}}\|v\|_{L^{2}\left(0, T_{0} ; D\left(\left(-A_{0}\right)^{\eta}\right)\right)}
$$

(by (2.10)), where $C_{T_{0}}<1$ for $T_{0}$ small enough. 
Then for integer $N$ we can define the maps $K_{0, J}, 1 \leq J \leq N$, by having $\forall \vec{v} \in L^{2}\left(\left(\frac{J-1}{N}\right) T, \frac{J}{N} T ; D\left(\left(-A_{0}\right)^{\eta}\right)\right)$,

$$
K_{0, J} \vec{v}(\cdot)=\int_{\frac{(J-1) T}{N}}^{(\cdot)} e^{A_{0}(\cdot-s)} C^{*} \int_{\frac{(J-1) T}{N}}^{s} e^{A_{1}(s-\tau)} C \vec{v}(\tau) d \tau d s .
$$

For $N$ large enough we will have from (2.15a) that $\left\|K_{0, J}\right\|<1 \forall J$, and we can hence use repeated iteration with the maps $K_{0, J}$ to show the bijectivity of $I-K_{0}$ on $L^{2}\left(0, T ; D\left(\left(-A_{0}\right)^{\eta}\right)\right)$, and the bounded invertibility of $I-K_{0}$ will follow from the Open Mapping Theorem.

We now use Proposition 2.2.1 to establish the crucial norm estimate which will ultimately validate the input operator $L$ (given in (2.2)).

Proposition 2.2.2. Let $\alpha$ be as specified in (1.1f); then:

(i) $\forall u \in U$ the function $e^{\mathcal{A}(\cdot)} \mathcal{B} u$ is an element in

$$
C\left([0, T] ; H_{1}\right) \times L^{2}\left(0, T ; D\left(\left(-A_{0}\right)^{\frac{1}{2}-\frac{\alpha}{4}}\right)\right) \cap_{\left(\frac{\alpha}{4}\right)} C\left([0, T] ; H_{0}\right),
$$

where $\forall \eta \in \mathbf{R},{ }_{\eta} C\left([0, T] ; H_{0}\right)$ is the Banach space

$$
\left\{\vec{v} \in C\left((0, T] ; H_{0}\right) \ni\|\vec{v}\|_{\eta} C\left((0, T] ; H_{0}\right)=\sup _{0<t \leq T} t^{\eta}\|\vec{v}(t)\|_{H_{0}}<\infty\right\} .
$$

(ii) $\forall 0<t \leq T, e^{\mathcal{A} t} \mathcal{B} \in \mathcal{L}\left(U, H_{1} \times H_{0}\right)$, and there exists a constant $\theta, 0<\theta<\frac{1}{2}$, such that the following norm estimate is satisfied:

$$
\left\|e^{\mathcal{A} t} \mathcal{B} u\right\|_{\mathcal{L}\left(U, H_{1} \times H_{0}\right)} \leq \frac{C_{0}}{t^{\theta}}
$$

where $C_{0}$ is some positive constant, and in fact $\theta \equiv \frac{\alpha}{4}$.

Proof of (i): If we consider, for fixed $u$, the quantity

$$
e^{\mathcal{A} t} \mathcal{B} u \equiv\left[\begin{array}{c}
z(t) \\
z_{t}(t) \\
v(t) \\
v_{t}(t)
\end{array}\right]=\left[\begin{array}{c}
\vec{z}(t) \\
\vec{v}(t)
\end{array}\right] \in\left[D\left(\mathcal{A}^{*}\right)\right]^{\prime},
$$

then necessarily $\vec{z}(\cdot)$ and $\vec{v}(\cdot)$ satisfy the coupled system,

$$
\begin{gathered}
\left\{\begin{array}{c}
z_{t t} \\
\frac{\partial z}{\partial \nu}=\left\{\begin{array}{cc}
v_{t} & \text { on } \Gamma_{0} \times(s, T) \\
0 & \text { on } \Gamma \backslash \Gamma_{0} \times(s, T) ; \\
z(0) & =z_{t}(0)=0
\end{array}\right.
\end{array}\right. \\
\left\{\begin{array}{l}
v_{t t}+\AA v+\AA v_{t}=-z_{t} \text { in }\left[D\left(\AA^{\frac{1}{2}}\right)\right]^{\prime} \\
v(0)=0 \\
v_{t}(0)=B u \text { in }\left[D\left(\AA^{\frac{1}{2}}\right)\right]^{\prime} ;
\end{array}\right.
\end{gathered}
$$


and by the uniqueness theory for ordinary differential equations, finding the solution of the above coupled system is tantamount to solving the following pair of coupled integral equations in $L^{2}\left(0, T ; H_{1} \times H_{0}\right)$ :

$$
\begin{aligned}
& \vec{z}(\cdot)=\int_{0}^{(\cdot)} e^{A_{1}(\cdot-\tau)} C \vec{v}(\tau) d \tau, \\
& \vec{v}(\cdot)=e^{A_{0}(\cdot)}\left[\begin{array}{c}
0 \\
B u
\end{array}\right]-\int_{0}^{(\cdot)} e^{A_{0}(\cdot-\tau)} C^{*} \vec{z}(\tau) d \tau .
\end{aligned}
$$

Formally then, (2.19) is equivalent to solving

$$
\vec{v}+K_{0} \vec{v}(\cdot)=e^{A_{0}(\cdot)}\left[\begin{array}{c}
0 \\
B u
\end{array}\right],
$$

where $K_{0}$ is as defined in $(2.5)$.

Now, considering the RHS of (2.20) we have by the definition of $B$ that

$$
[0, B]^{T} \in \mathcal{L}\left(U, D\left(\AA^{\frac{1}{2}}\right) \times H^{-\alpha}\left(\Gamma_{0}\right)\right),
$$

and from $(1.10) H_{0}^{\alpha}\left(\Gamma_{0}\right)=D\left(\AA^{\frac{\alpha}{4}}\right)$; so we can deduce from Theorem A (i)-(ii) that

$$
\begin{aligned}
e^{A_{0}(\cdot)}\left[\begin{array}{c}
0 \\
B
\end{array}\right] & =\left(-A_{0}\right)^{\frac{\alpha}{4}} e^{A_{0}(\cdot)}\left(-A_{0}\right)^{-\frac{\alpha}{4}}\left[\begin{array}{c}
0 \\
B
\end{array}\right] \\
& \in \mathcal{L}\left(U, L^{2}\left(0, T ; D\left(\left(-A_{0}\right)^{\frac{1}{2}-\frac{\alpha}{4}}\right)\right)\right) .
\end{aligned}
$$

As $\frac{1}{2}-\frac{\alpha}{4}=\zeta$ - where $\zeta=\frac{1}{16}$ if $\Omega$ is a rectangle, and $\zeta=\frac{1}{12}$ if boundary $\Gamma$ is smoothwe can hence apply the inverse $\left(I+K_{0}\right)^{-1}$, the existence of which is ensured by Proposition 2.2 .1 , to $(2.20)$ to obtain

$$
\left[\begin{array}{c}
v(\cdot) \\
v_{t}(\cdot)
\end{array}\right] \equiv \vec{v}(\cdot)=\left(I+K_{0}\right)^{-1} e^{A_{0}(\cdot)}\left[\begin{array}{c}
0 \\
B u
\end{array}\right] \in L^{2}\left(0, T ; D\left(\left(-A_{0}\right)^{\frac{1}{2}-\frac{\alpha}{4}}\right)\right) .
$$

Going back to variable $\vec{z}$, defining

$$
\left[\begin{array}{c}
z(\cdot) \\
z_{t}(\cdot)
\end{array}\right]=\vec{z}(\cdot) \equiv \int_{0}^{(\cdot)} e^{A_{1}(\cdot-\tau)} C \vec{v}(\tau) d \tau
$$

will yield a fortiori via Theorem 2.B - which again is directly applicable here as $\frac{1}{3} \leq 2-\alpha<\frac{1}{2}$ in the case of a general smooth domain, and $\frac{1}{4} \leq 2-\alpha<\frac{1}{2}$ if $\Omega$ is a rectangle-the unique (weak) solution of (2.19a), and consequently we have that

$$
\vec{z} \in C\left([0, T] ; H_{1}\right) .
$$

In addition, as $A_{0}$ is the direct sum of two normal operators (see [7], p.290), we have by the analyticity of $A_{0}$ that

$$
K_{0} \in \mathcal{L}\left(L^{2}\left(0, T ; D\left(\left(-A_{0}\right)^{\frac{1}{2}-\frac{\alpha}{4}}\right), C\left([0, T] ; H_{0}\right)\right),\right.
$$


and we hence deduce from the integral representation of $\vec{v}(\cdot)$ in $(2.19 \mathrm{~b})$ that

$$
\vec{v}(\cdot) \in_{\left(\frac{\alpha}{4}\right)} C\left([0, T] ; H_{0}\right),
$$

where the singularity at 0 is of course due to the presence of the unbounded operator $B$. So, defining

$$
e^{\mathcal{A}(\cdot)} \mathcal{B} u \equiv\left[\begin{array}{c}
\vec{z}(\cdot) \\
\vec{v}(\cdot)
\end{array}\right]
$$

where $[\vec{z}, \vec{v}]$ solves $(2.19)$, will yield the result in (i) via the regularity properties (2.23), $(2.25)$, and $(2.26 \mathrm{~b})$.

(ii): With $[\vec{z}, \vec{v}]$ as defined by $(2.27)$, we use the a posteriori estimates given from the regularity result Theorem $2 . \mathrm{B}$ and the inversion of the operator $I-K_{0}$ to majorize $\|\vec{z}\|_{C\left([0, T] ; H_{1}\right)}$, where $\vec{z}$ is as given in (2.24); namely, we have

$$
\begin{aligned}
& \|\vec{z}\|_{C\left([0, T] ; H_{1}\right)} \leq C_{1}\left\|v_{t}\right\|_{L^{2}\left(0, T ; H^{2-\alpha}\left(\Gamma_{0}\right)\right)} \\
& \leq C_{2}\left\|\left(I+K_{0}\right)^{-1}\right\|_{\mathcal{L}\left(L^{2}\left(0, T ; D\left(\left(-A_{0}\right)^{\frac{1}{2}-\frac{\alpha}{4}}\right)\right)\right.} \\
& \cdot\left\|e^{A_{0}(\cdot)}\left[\begin{array}{c}
0 \\
B u
\end{array}\right]\right\|_{\left.L^{2}\left(0, T ; D\left(-A_{0}\right)^{\frac{1}{2}-\frac{\alpha}{4}}\right)\right)} \\
& \leq C_{3}\left\|\left(-A_{0}\right)^{-\frac{\alpha}{4}}\left[\begin{array}{c}
0 \\
B u
\end{array}\right]\right\|_{H_{0}} \quad \text { from Theorem 2.A(ii) } \\
& \leq C_{4}\|u\|_{U} .
\end{aligned}
$$

Moreover, using the representation for $\vec{v}(\cdot)$ in $(2.19 \mathrm{~b})$ and Proposition (2.2.3), we obtain, upon taking norms, the pointwise estimate (valid on $(0, T]$ because of the regularity given in $(2.26 \mathrm{~b}))$,

$$
\|\vec{v}(t)\|_{H_{0}} \leq\left\|e^{A_{0} t}\left[\begin{array}{c}
0 \\
B u
\end{array}\right]\right\|_{H_{0}}+\left\|v_{t}\right\|_{L^{2}\left(0, T ; H^{2-\alpha}\left(\Gamma_{0}\right)\right)}
$$

(after using the analyticity of $A_{0}$ and the continuity of the trace map provided in Theorem 2.B)

$$
\leq\left\|e^{A_{0} t}\left[\begin{array}{c}
0 \\
B u
\end{array}\right]\right\|_{H_{0}}+C\|u\|_{U}
$$

Moreover, we have again from the analyticity of $A_{0}$ and Theorem 2.A(ii),

$$
\begin{aligned}
\left\|e^{A_{0} t}\left[\begin{array}{c}
0 \\
B u
\end{array}\right]\right\|_{H_{0}} & =\left\|\left(-A_{0}\right)^{\frac{\alpha}{4}} e^{A_{0} t}\left(-A_{0}\right)^{-\frac{\alpha}{4}}\left[\begin{array}{c}
0 \\
B u
\end{array}\right]\right\|_{H_{0}} \\
& \leq \frac{C_{T}}{t^{\frac{\alpha}{4}}}\|u\|_{U} .
\end{aligned}
$$

Coupling (2.28) and ((2.29)-(2.30), we indeed obtain the desired estimate

$$
\left\|e^{\mathcal{A} t} \mathcal{B} u\right\|_{\substack{H_{1} \times H_{0} \\ 13}} \leq \frac{C}{t^{\theta}}\|u\|_{U},
$$


where $\theta \equiv \frac{\alpha}{4}$, and Proposition (2.2.2)(ii) is proved.

Proof of Lemma 2.1: This is a simple consequence of Proposition 2.2.2. Indeed, from (2.2), we have $\forall u \in L^{2}(0, T ; U)$

$$
\begin{aligned}
\|L u(t)\|_{H_{1} \times H_{0}} & \leq \int_{0}^{t}\left\|e^{\mathcal{A}(t-s)} \mathcal{B} u(s)\right\|_{H_{1} \times H_{0}} d s \\
& \leq \int_{0}^{t} \frac{1}{(t-s)^{\frac{\alpha}{4}}}\|u(s)\|_{U} d s \\
& \leq C_{T}\|u\|_{L_{2}(0, T ; U)},
\end{aligned}
$$

after using estimate (2.17) of Proposition 2.2.2(ii) which implies $\|L u(t)\|_{L^{\infty}\left(0, T ; H_{1} \times H_{0}\right)} \leq$ $C_{T}\|u\|_{L^{2}(0, T ; U)}$. The usual density argument, together with the completeness of $C([0, T] ; U)$, proves continuity in time as asserted in Lemma 2.1.

In the sequel we shall need properties of the map $L_{s}: L^{2}(s, T ; U) \rightarrow L^{2}\left(s, T ; H_{1} \times H_{0}\right)$ defined as

$$
\left(L_{s} u\right)(t) \equiv \int_{s}^{t} e^{\mathcal{A}(t-\tau)} \mathcal{B} u(\tau) d \tau
$$

where $s<t<T$.

It is now a straightforward result-via Lemma 2.1-that the continuity of the map $L_{s}$ : $L^{2}(s, T ; U) \rightarrow C\left([s, T] ; H_{1} \times H_{0}\right)$ is preserved uniformly in the parameter " $s$ ". In fact, we have the following:

\section{Corollary 2.2.1.}

(i) The operator $L_{s}$, defined on $U, \forall 0<s<T$, given by

$$
L_{s} u(\cdot) \equiv \int_{s}^{(\cdot)} e^{\mathcal{A}(\cdot-\tau)} \mathcal{B} u(\tau) d \tau
$$

is an element of $\mathcal{L}\left(L^{2}(s, T ; U), C\left([s, T] ; H_{1} \times H_{0}\right)\right.$ with a norm uniform in $s$.

(ii) The unique solution to (1.15), for fixed $u \in L^{2}(s, T ; U)$, is given by

$$
\left[\begin{array}{c}
\vec{z}(\cdot) \\
\vec{v}(\cdot)
\end{array}\right] \equiv Y(\cdot)=e^{\mathcal{A}(\cdot-s)} Y_{0}+L_{s} u(\cdot) \in C\left([s, T] ; H_{1} \times H_{0}\right) .
$$

(iii) The adjoint operator

$$
L_{s}^{*} \in \mathcal{L}\left(L^{2}\left(s, T ; H_{1}^{*} \times H_{0}^{*}\right), L^{2}(s, T ; U)\right)
$$

is given by

$$
L_{s}^{*} \hat{Y}(\cdot)=\int_{(\cdot)}^{T} \mathcal{B}^{*} e^{\mathcal{A}^{*}(\tau-\cdot)} \hat{Y}(\tau) d \tau=\mathcal{B}^{*} \int_{(\cdot)}^{T} e^{\mathcal{A}^{*}(\tau-\cdot)} \hat{Y}(\tau) d \tau .
$$

Proof: Given the norm estimate (2.17) the proofs of (i)-(iii) are straightforward and may be omitted. Moreover, it can readily be shown that $\forall \hat{Y} \in L^{2}\left(s, T ; D\left(\mathcal{A}^{*}\right)\right)$,

$$
L_{s}^{*} \hat{Y}(\cdot)=\mathcal{B}^{*} \int_{(\cdot)}^{T} e^{\mathcal{A}^{*}(\tau-\cdot)} \hat{Y}(\tau) d \tau
$$


so $L_{s}^{*}$ can be considered as an extension by density of the operator

$$
\hat{Y}(\cdot) \rightarrow \mathcal{B}^{*} \int_{(\cdot)}^{T} e^{\mathcal{A}^{*}(\tau-\cdot)} \hat{Y}(\tau) d \tau, \quad \forall \hat{Y}(\cdot) \in L^{2}\left(s, T ; D\left(\mathcal{A}^{*}\right)\right) ;
$$

hence the equality in (2.37) is entirely justified.

2.2. The Associated Optimal Control Problem: Proof of Theorem 1.1. Given the existence and uniqueness established in Section 2.2 for the solution $[\vec{z}, \vec{v}]^{T}=Y$ of (1.15), we can proceed to consider the optimal control problem for $0 \leq s<T$,

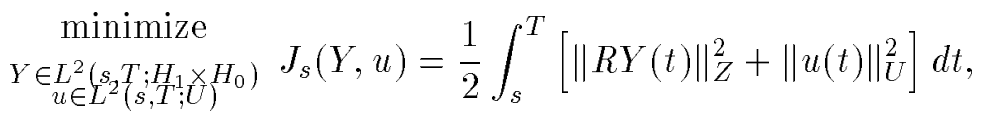

subject to $Y(\cdot)=e^{\mathcal{A}(\cdot-s)} Y_{0}+L_{s} u(\cdot)$, where $Y_{0}$ is the initial data

$$
\left[z_{0}, z_{1}, v_{0}, v_{1}\right]^{T} \in H_{1} \times H_{0} \text {. }
$$

Lemma 2.3.1. There exists a unique optimal pair

$$
\begin{aligned}
& {\left[\vec{z}^{*}\left(\cdot, s ; Y_{0}\right), \vec{v}^{*}\left(\cdot, s ; Y_{0}\right), u^{*}\left(\cdot, s ; Y_{0}\right)\right]^{T}} \\
& \quad=\left[Y^{*}\left(\cdot, s ; Y_{0}\right), u^{*}\left(\cdot, s ; Y_{0}\right)\right]^{T} \in C\left([s, T] ; H_{1} \times H_{0}\right) \times L^{2}(s, T ; U),
\end{aligned}
$$

which solves (2.38) and which is given explicitly by

$$
\begin{gathered}
u^{*}\left(\cdot, s ; Y_{0}\right)=-\left(I_{s}+L_{s}^{*} R^{*} R L_{s}\right)^{-1} L_{s}^{*} R^{*} R e^{\mathcal{A}(\cdot-s)} Y_{0} ; \\
{\left[\begin{array}{c}
\vec{z}^{*}\left(\cdot, s ; Y_{0}\right) \\
\vec{v}^{*}\left(\cdot, s ; Y_{0}\right)
\end{array}\right] \equiv Y^{*}\left(\cdot, s ; Y_{0}\right)=e^{\mathcal{A}(\cdot-s)} Y_{0}+L_{s} u^{*}\left(\cdot, s ; Y_{0}\right)}
\end{gathered}
$$

(where $\left(I_{s}+L_{s}^{*} R^{*} R L_{s}\right)^{-1} \in \mathcal{L}\left(L^{2}(0, T ; U)\right.$; here $I_{s}+L_{s}^{*} R^{*} R L_{s}$ is the identity map on $L^{2}(0, T ; U)$ )

Proof: If we define $V \in \mathcal{L}\left(L^{2}\left(s, T ; H_{1} \times H_{0}\right) \times L^{2}(s, T ; U), L^{2}\left(s, T ; H_{1} \times H_{0}\right)\right)$ by having $\forall[Y, u]^{T} \in L^{2}\left(s, T ; H_{1} \times H_{0}\right) \times L^{2}(s, T ; U)$,

$$
V(Y, u)=Y(\cdot)-e^{\mathcal{A}(\cdot-s)} Y_{0}-\int_{0}^{(\cdot)} e^{\mathcal{A}(\cdot-\tau)} \mathcal{B} u(\tau) d \tau
$$

(see Lemma 2.1 and Corollary 2.2.1); we then have that (2.38) is equal to the following optimization problem:

$$
\text { minimize } \underset{\substack{Y \in L^{2}\left(s_{2} T ; H_{1} \times H_{0}\right) \\ u \in L^{2}(s, T ; \cup)}}{J(Y, u)}=\frac{1}{2} \int_{s}^{T}\|R Y(t)\|_{Z}^{2}+\|u(t)\|_{U}^{2} d t,
$$

subject to $V(Y, u)=0$.

Subsequently, by the standard theory of convex optimization (see [5, p.35]), there exists a unique pair

$$
\begin{aligned}
& {\left[Y^{*}\left(\cdot, s ; Y_{0}\right), u^{*}\left(\cdot, s ; Y_{0}\right)\right]^{T}} \\
& \quad=\left[\vec{z}^{*}\left(\cdot, s ; Y_{0}\right), \vec{v}^{*}\left(\cdot, s ; Y_{0}\right), u^{*}\left(\cdot, s ; Y_{0}\right)\right] \in L^{2}\left(s, T ; H_{1} \times H_{0}\right) \times L^{2}(s, T ; U),
\end{aligned}
$$


which solves (2.41), and from Corollary 2.2.1(ii) $Y^{*}\left(\cdot, ; Y_{0}\right) \in C\left([s, T] ; H_{1} \times H_{0}\right)$. Moreover, the Frechét derivative of $V$ can easily be computed at every $[Y, u]^{T} \in L^{2}\left(s, T ; H_{1} \times H_{0}\right) \times$ $L^{2}(s, T ; U)$ as the "constant" $V^{\prime}$, where $V^{\prime}$ is defined by having for every $[Y, u]^{T}$,

$$
V^{\prime}\left[\begin{array}{l}
Y \\
u
\end{array}\right]=\left[\begin{array}{cc}
I_{s} & 0 \\
0 & -L_{s}
\end{array}\right]\left[\begin{array}{l}
Y \\
u
\end{array}\right]
$$

(here $I_{s}$ is the identity on $\left.L^{2}\left(s, T ; H_{1} \times H_{0}\right)\right)$. $V^{\prime}$ is evidently surjective; to wit $\forall Y \in$ $L^{2}\left(s, T ; H_{1} \times H_{0}\right), V^{\prime}\left[\begin{array}{c}Y \\ 0\end{array}\right]=Y$, so by Liusternik's Lagrange Multiplier Theorem (see [18, p.243]) there exist $\mathrm{s} \lambda^{*} \in L^{2}\left(s, T ; H_{1}^{*} \times H_{0}^{*}\right)$ such that

$$
J^{\prime}\left(Y^{*}\left(\cdot, s ; Y_{0}\right), u^{*}\left(\cdot, s ; Y_{0}\right)\right)+\left\langle\lambda^{*}, V^{\prime}\left[\begin{array}{l}
\cdot \\
\cdot
\end{array}\right]\right\rangle=0
$$

(where $\langle\cdot, \cdot\rangle$ denotes the duality pairing between $L^{2}\left(s, T ; H_{1} \times H_{0}\right)$ and its dual); i.e. $\forall[Y, u]^{T} \in L^{2}\left(s, T ; H_{1} \times H_{0}\right) \times L^{2}(s, T ; U)$,

$$
\begin{aligned}
0=\left\langle R^{*} R Y^{*}(\cdot\right. & \left.\left., s ; Y_{0}\right), Y\right\rangle+\left\langle\lambda^{*}, Y-L_{s} u\right\rangle \\
& +\left(u^{*}\left(\cdot, s ; Y_{0}\right), u\right)_{L^{2}(s, T ; U)} .
\end{aligned}
$$

We thus deduce from (2.43) that

$$
\begin{aligned}
R^{*} R Y^{*}\left(\cdot, s ; Y_{0}\right) & =-\lambda^{*} ; \\
u^{*}\left(\cdot, s ; Y_{0}\right) & =L_{s}^{*} \lambda^{*}=-L_{s}^{*} R^{*} R Y^{*}\left(\cdot, s ; Y_{0}\right) ; \\
Y^{*}\left(\cdot, s ; Y_{0}\right) & =e^{\mathcal{A}(\cdot-s)} Y_{0}+L_{s} u^{*}\left(\cdot, s ; Y_{0}\right) .
\end{aligned}
$$

From here, (2.45) yields that

$$
u^{*}\left(\cdot, s ; Y_{0}\right)=-L_{s}^{*} R^{*} R e^{\mathcal{A}(\cdot-s)} Y_{0}-L_{s}^{*} R^{*} R L_{s} u^{*}\left(\cdot, s ; Y_{0}\right),
$$

or

$$
u^{*}\left(\cdot, s ; Y_{0}\right)=-\left(I_{s}+L_{s}^{*} R^{*} R L_{s}\right)^{-1} L_{s}^{*} R^{*} R e^{\mathcal{A}(\cdot-s)} Y_{0},
$$

using the fact that $I_{s}+L_{s}^{*} R^{*} R L_{s}$ is invertible on $L^{2}(s, T ; U)$ by Lax-Milgram, together with regularity result of Corollary 2.2.1 (and of course, where $I_{s}$ is this time the identity on $\left.L^{2}(s, T ; U)\right)$.

2.3. Additional Regularity Properties of the Optimal Solution. In this section we show that the optimal control $u^{*}\left(\cdot, s ; Y_{0}\right)$, as given in Lemma 2.3.1, is actually continuous in time, and that $\vec{v}^{*}\left(\cdot, s ; Y_{0}\right)$ of $(2.39 \mathrm{~b})$ has greater regularity in the spatial variable then previously stated. These properties are critical for the derivation of the Riccati Equation.

Proposition 2.4.1. Let $\vec{v}(\cdot)$ be as in (2.36); then $\vec{v} \in L^{2}\left(s, T ;\left[D\left(\AA^{\frac{1}{2}}\right)\right]^{2}\right)$. 
Proof: WLOG we can take $s \equiv 0$. Finding the solution $Y(\cdot)=\left[\begin{array}{c}\vec{z}(\cdot) \\ \vec{v}(\cdot)\end{array}\right]$ of $(1.15)$ is formally tantamount to solving the coupled system of integral equations

$$
\begin{aligned}
& \vec{z}(t)=e^{A_{1} t} z_{0}+\int_{0}^{t} e^{A_{1}(t-\tau)} C \vec{v}(\tau) d \tau \\
& \vec{v}(t)=e^{A_{0} t} v_{0}-\int_{0}^{t} e^{A_{0}(t-\tau)} C^{*} \vec{z}(\tau) d \tau+\int_{0}^{t} e^{A_{0}(t-\tau)}\left[\begin{array}{c}
0 \\
B u(\tau)
\end{array}\right] d \tau,
\end{aligned}
$$

which is formally equivalent to solving

$$
\vec{v}(t)+K_{0} \vec{v}(t)=e^{A_{0} t} v_{0}-\int_{0}^{t} e^{A_{0}(t-\tau)} C^{*} e^{A_{1} \tau} z_{0} d \tau+\int_{0}^{t} e^{A_{0}(t-\tau)}\left[\begin{array}{c}
0 \\
B u(\tau)
\end{array}\right] d \tau,
$$

where $K_{0}$ is as defined in (2.5). Now by [19]

$$
C^{*} e^{A_{1}(\cdot)} z_{0} \in L^{2}\left(0, T ; D\left(\AA^{\frac{1}{2}}\right) \times H^{-\frac{1}{2}}\left(\Gamma_{0}\right)\right),
$$

so we can use this fact along with the same argument used in establishing (2.14) of Proposition 2.2.1(i) to have that the RHS of (2.49) is in

$$
L^{2}\left(0, T ; D\left(\left(-A_{0}\right)^{\frac{1}{2}}\right)\right) .
$$

Consequently by this same lemma we can apply the inverse $\left(I+K_{0}\right)^{-1}$ to both sides of $(2.48)$ to obtain

$$
\begin{aligned}
{\left[\begin{array}{c}
v \\
v_{t}
\end{array}\right]=\vec{v}(\cdot)=} & \left(I+K_{0}\right)^{-1}\left[e^{A_{0}(\cdot)} v_{0}-\int_{0}^{(\cdot)} e^{A_{0}(\cdot-\tau)} C^{*} e^{A_{1} \tau} z_{0} d \tau\right. \\
& \left.+\int_{0}^{(\cdot)} e^{A_{0}(\cdot-\tau)}\left[\begin{array}{c}
0 \\
B u(\tau)
\end{array}\right] d \tau\right] \in L^{2}\left(0, T ;\left[D\left(\AA^{\frac{1}{2}}\right)\right]^{2}\right),
\end{aligned}
$$

after using Theorem 2.A(i). We can subsequently derive the solution $\vec{z}(\cdot)$ of (2.47a) by setting

$$
\begin{aligned}
{\left[\begin{array}{c}
z \\
z_{t}
\end{array}\right]=\vec{z}(\cdot) \equiv } & e^{A_{1}(\cdot)} v_{0}+\int_{0}^{(\cdot)} e^{A_{1}(\cdot-\tau)} C\left[\left(I+K_{0}\right)^{-1} e^{A_{0}(\cdot)} v_{0}\right]_{\tau} d \tau \\
& -\int_{0}^{(\cdot)} e^{A_{1}(\cdot-\tau)} C\left[\left(I+K_{0}\right)^{-1} \int_{0}^{(\cdot)} e^{A_{0}(\cdot-\sigma)} C^{*} e^{A_{1} \sigma} z_{0} d \sigma\right]_{\tau} d \tau \\
& +\int_{0}^{(\cdot)} e^{A_{1}(\cdot-\tau)} C\left[\left(I+K_{0}\right)^{-1} \int_{0}^{(\cdot)} e^{A_{0}(\cdot-\sigma)}\left[\begin{array}{c}
0 \\
B u(\sigma)
\end{array}\right] d \sigma\right]_{\tau} d \tau ;
\end{aligned}
$$


and $z$ is in $C\left([0, T] ; H_{1}\right)$ by Theorem $2 . \mathrm{B}$ which is directly applicable, as we can readily compute that the solution of $(2.47 \mathrm{a})$ which we have here obtained satisfies

$$
\begin{aligned}
z_{t t} & =\Delta z \\
\frac{\partial z}{\partial \nu} & =\left\{\begin{array}{rr}
v_{t} & \text { on } \Gamma_{0} \times(0, T) \\
0 & \text { on } \Gamma \backslash \Gamma_{0} \times(0, T) .
\end{array} ;\right. \\
z(0) & =z_{t}(0)=0 .
\end{aligned}
$$

A fortiori then, the solution $[\vec{z}, \vec{v}]^{T}$ defined in (2.51)-(2.52) solves the ODE (1.15), and a uniqueness argument concludes the proof.

Remark 2.4. As we have just shown in Proposition 2.4.1 that every $\vec{v}(\cdot)$ of $(2.36)$ (corresponding to some fixed $\left.u \in L^{2}(0, T ; U)\right)$ is in $L^{2}\left(s, T ;\left[D\left(\AA^{\frac{1}{2}}\right)\right]^{2}\right)$, then in particular, the optimal $\vec{v}^{*}\left(\cdot, s ; Y_{0}\right)$ given by $(2.39 \mathrm{~b})$ has the stated regularity.

Proposition 2.4.2. Let $L_{s}^{*}$ be as given in (2.37); then

$$
L_{s}^{*} \in \mathcal{L}\left(C\left([s, T] ; H_{1}^{*} \times H_{0}^{*}\right), C([s, T] ; U)\right),
$$

and $\forall \hat{Y} \in C\left([s, T] ; H_{1}^{*} \times H_{0}^{*}\right)$ we have the estimate

$$
\left\|L_{s}^{*} \hat{Y}\right\|_{C([s, T] ; U)} \leq C_{T}\|\hat{Y}\|_{C\left([s, T] ; H_{1}^{*} \times H_{0}^{*}\right)},
$$

where $C_{T}$ is independent of $s, 0 \leq s<T$.

Proof: $L_{s}^{*} \hat{Y}$ is easily seen to be in $C([s, T) ; U) \forall \hat{Y}$ in $C\left([s, T) ; H_{1}^{*} \times H_{0}^{*}\right)$, and hence we need only show that there is no singularity at the endpoint $T$. So $\forall t$, $s \leq t<T$, we have by $(2.17)$,

$$
\begin{aligned}
\left\|L_{s}^{*} \hat{Y}(t)\right\|_{U} & \leq \int_{t}^{T}\left\|B^{*} e^{\mathcal{A}^{*}(\tau-t)} \hat{Y}(\tau)\right\|_{U} d \tau \\
& =\int_{t}^{T} \frac{C_{0}}{(\tau-t)^{\theta}}\|\hat{Y}(\tau)\|_{H_{1}^{*} \times H_{0}^{*}} d \tau \\
& \leq \frac{C_{0}(T-s)^{1-\theta}}{1-\theta}\|\hat{Y}\|_{C\left([s, T] ; H_{1}^{*} \times H_{0}^{*}\right)},
\end{aligned}
$$

where $\theta$ is as in (2.17), and we have the result.

Proposition 2.4.3. $L_{s} \in \mathcal{L}\left(C([s, T] ; U), C\left([s, T] ; H_{1} \times H_{0}\right)\right.$ and $\forall u \in C([s, T] ; U)$ we have the estimate

$$
\left\|L_{s} u(t)\right\|_{H_{1} \times H_{0}} \leq C_{T}\|u\|_{C([s, T] ; U)},
$$

where $C_{T}$ is independent of $s, 0 \leq s<T$.

Proof: Similar to that of Proposition 2.4.2. 
Lemma 2.4.1. The operator $I_{s}+L_{s}^{*} R^{*} R L_{s}$ is boundedly invertible on $C([s, T] ; U)$ with the following norm estimate:

$$
\left\|\left(I_{s}+L_{s}^{*} R^{*} R L_{s}\right)^{-1}\right\|_{\mathcal{L}(C([s, T] ; U))} \leq C_{T},
$$

where $C_{T}$ is independent of $s, 0 \leq s<T$.

Proof: We make use here of a bootstrap argument: For an arbitrary $g \in C([s, T] ; U)$ we wish to show that there exists a unique $f \in C([s, T] ; U)$ such that

$$
f+L_{s}^{*} R^{*} R L_{s} f=g \text { in } C([s, T] ; U) .
$$

As $I_{s}+L_{s}^{*} R^{*} R L_{s}$ is invertible in $L^{2}(s, T ; U)$ (by Lax-Milgram), then certainly there exists a unique $h \in L^{2}(s, T ; U)$ such that

$$
h+L_{s}^{*} R^{*} R L_{s} h=L_{s}^{*} R^{*} R L_{s} g \text { in } L^{2}(s, T ; U) .
$$

Thus, by Propositions (2.4.2)-(2.4.3),

$$
h=L_{s}^{*} R^{*} R L_{s} g-L_{s}^{*} R^{*} R L_{s} h \text { in } C([s, T] ; U),
$$

and it is easy to see that $f \equiv g-h \in C([s, T] ; U)$ will be the unique solution to (2.58). The result then follows upon application of the Open Mapping Theorem. The norm estimate (2.57) is a consequence of the estimates given in (2.54) and (2.56).

Corollary 2.4.1. $u^{*}\left(\cdot, s ; Y_{0}\right)$, given by (2.39a) is in $C([s, T] ; U)$.

Proof: By Proposition 2.4.2, $L_{s}^{*} R^{*} R e^{\mathcal{A}(\cdot)} Y_{0} \in C([s, T] ; U)$, and the result follows from Lemma 2.4.1.

Corollary 2.4.2. We have the following estimates for the optimal pair:

(i) $\left\|\vec{v}^{*}\left(\cdot, s ; Y_{0}\right)\right\|_{L^{2}\left(s, T ;\left[D\left(\AA^{\frac{1}{2}}\right)\right]^{2}\right)} \leq C_{T}\left\|Y_{0}\right\|_{H_{1} \times H_{0}}$;

(ii) $\left\|u^{*}\left(\cdot, s ; Y_{0}\right)\right\|_{C\left([s, T] ; U_{0}\right)} \leq C_{T}\left\|Y_{0}\right\|_{H_{1} \times H_{0}}$;

(iii) $\left\|Y^{*}\left(\cdot, s ; Y_{0}\right)\right\|_{C\left([s, T] ; H_{1} \times H_{0}\right)} \leq C_{T}\left\|Y_{0}\right\|_{H_{1} \times H_{0}}$.

Proof: Estimates (ii), (iii) follow from (2.45b-c) and (2.46b), and the results of Propositions 2.4.2-2.4.3 and Lemma 2.4.1. For the component $\vec{v}^{*}\left(\cdot, s ; Y_{0}\right)$ we have from $(2.51)$ that

$$
\begin{aligned}
\vec{v}^{*}\left(\cdot, s ; Y_{0}\right) & =\left(I+K_{0}\right)^{-1}\left[e^{A_{0}(\cdot-s)} v_{0}-\int_{s}^{(\cdot)} e^{A_{0}(\cdot-\tau)} C^{*} e^{A_{1}(\tau-s)} z_{0} d \tau\right. \\
& \left.+\int_{s}^{(\cdot)} e^{A_{0}(\cdot-\tau)}\left[\begin{array}{c}
0 \\
B u^{*}\left(\tau, s ; Y_{0}\right)
\end{array}\right] d \tau\right],
\end{aligned}
$$

where equality can be taken in $L^{2}\left(s, T ;\left[D\left(\AA^{\frac{1}{2}}\right)\right]^{2}\right)$. Now for the RHS of $(2.64)$ :

$$
\begin{aligned}
\left\|\left(I+K_{0}\right)^{-1} e^{A_{0}(\cdot-s)} v_{0}\right\|_{L^{2}\left(s, T ;\left[D\left(\AA^{\frac{1}{2}}\right)\right]^{2}\right.} & \leq C_{T}\left\|e^{A_{0}(\cdot-s)} v_{0}\right\|_{L^{2}\left(s, T ;\left(D\left(-A_{0}\right)^{\frac{1}{2}}\right)\right)} \\
& \leq C_{T}\left\|v_{0}\right\|_{H_{0}},
\end{aligned}
$$


after making use of the continuity of the map $\left(I+K_{0}\right)^{-1}$ and Theorem A(i)-(ii).

Again from [19], $C^{*} e^{A_{1}(\cdot-s)} z_{0} \in L^{2}\left(s, T ; D\left(\AA^{\frac{1}{2}}\right) \times H^{-\frac{1}{2}}\left(\Gamma_{0}\right)\right)$, and so we will have

$$
\begin{aligned}
\left\|\left(I+K_{0}\right)^{-1}\left[\int_{s}^{(\cdot)} e^{A_{0}(\cdot-\tau)} C^{*} e^{A_{1}(\tau-s)} z_{0} d \tau\right]\right\|_{L^{2}\left(s, T ;\left[D\left(\AA^{\frac{1}{2}}\right)\right]^{2}\right)} \\
\leq C_{T}\left\|\int_{s}^{(\cdot)} e^{A_{0}(\cdot-\tau)} C^{*} e^{A_{1}(\tau-s)} z_{0} d \tau\right\|_{L^{2}\left(s, T ;\left[D\left(\AA^{\frac{1}{2}}\right)\right]^{2}\right)} \\
\leq\left\|C^{*} e^{A_{1}(\cdot-s)} z_{0}\right\|_{L^{2}\left(s, T ; D\left(\AA^{\frac{1}{2}}\right) \times H^{-\frac{1}{2}}\left(\Gamma_{0}\right)\right)} \\
\leq C_{T}\left\|z_{0}\right\|_{H_{1}},
\end{aligned}
$$

after using the theory for analytic semigroups followed by the regularity estimate in [19]. Likewise, by the theory for analytic semigroups we obtain the estimate

$$
\left\|\int_{s}^{(\cdot)} e^{A_{0}(\cdot-\tau)}\left[\begin{array}{c}
0 \\
B u^{*}\left(\tau, s ; Y_{0}\right)
\end{array}\right] d \tau\right\|_{L^{2}\left(s, T ;\left[D\left(\AA^{\frac{1}{2}}\right)\right]^{2}\right)} \leq C_{T}\left\|u^{*}\left(\cdot, s ; Y_{0}\right)\right\|_{L^{2}(s, T ; U)},
$$

and using the characterization of $u^{*}\left(\cdot, s ; Y_{0}\right)$ in $(2.46 \mathrm{~b})$, we consequently have from Proposition 2.4.3 and (2.67) that

$$
\left\|\int_{s}^{(\cdot)} e^{A_{0}(\cdot-\tau)}\left[\begin{array}{c}
0 \\
B u^{*}\left(\tau, s ; Y_{0}\right)
\end{array}\right] d \tau\right\|_{L^{2}\left(s, T ;\left[D\left(\AA^{\frac{1}{2}}\right)\right]^{2}\right)} \leq C_{T}\left\|Y_{0}\right\|_{H_{1} \times H_{0}} .
$$

Thus, combining (2.64)-(2.66) and (2.68) we arrive at

$$
\left\|\vec{v}^{*}\left(\cdot, s ; Y_{0}\right)\right\|_{L^{2}\left(s, T ;\left[D\left(\AA^{\frac{1}{2}}\right)\right]^{2}\right)} \leq C_{T}\left\|Y_{0}\right\|_{H_{1} \times H_{0}},
$$

and our proof of (i) is concluded.

Remark 2.5. The proof of Theorem 1.1 follows from Lemma 2.3.1, Proposition 2.4.1, and Corollaries 2.4.1 and 2.4.2.

\section{The Riccati Operator}

3.1. Definition of the Operator $\Phi(\cdot, \cdot)$. At this point we introduce the (evolution) operator $\Phi(t, s)$ defined by

$$
\Phi(t, s) Y_{0}=Y^{*}\left(t, s ; Y_{0}\right), \quad Y_{0} \in H_{1} \times H_{0}, \quad 0 \leq s \leq t \leq T,
$$

and one can readily establish that

$$
\begin{gathered}
\Phi(t, t) Y_{0}=Y_{0} \\
\Phi(t, \tau) \Phi(\tau, s)=\Phi(t, s), \quad 0 \leq s \leq \tau \leq t \leq T .
\end{gathered}
$$

Furthermore, one has

Proposition 3.1.1. With $\Phi(\cdot, \cdot)$, as defined in (3.1), the following properties hold: 
(i) For fixed $s, 0 \leq s \leq T$, the map $t \rightarrow \Phi(t, s) Y_{0}$ is continuous in $H_{1} \times H_{0}$, $s \leq t \leq T$

(ii) for fixed $t, s \leq t \leq T$ and $Y_{0} \in H_{1} \times H_{0}$, the map $s \rightarrow \Phi(t, s) Y_{0}$ is continuous in $H_{1} \times H_{0}$.

Proof. Having established regularity properties of Corollary 2.4.2, the proof is the same as in [10] and is hence omitted.

With $\Phi(\cdot, \cdot)$ defined as in $(3.1)$, we subsequently define

$$
P(t) Y_{0}=\int_{t}^{T} e^{\mathcal{A}^{*}(\tau-t)} R^{*} R \Phi^{*}\left(\tau, t ; Y_{0}\right) d \tau
$$

and a fortiori $P(t) \in \mathcal{L}\left(H_{1} \times H_{0}, H_{1}^{*} \times H_{0}^{*}\right) \quad \forall t, 0 \leq t \leq T$, and

$$
P(\cdot) \in \mathcal{L}\left(H_{1} \times H_{0}, L^{\infty}\left(0, T ; H_{1}^{*} \times H_{0}^{*}\right)\right) .
$$

Again, in a standard way we can show that:

Proposition 3.1.2. $P(\cdot) \in \mathcal{L}\left(H_{1} \times H_{0}, C\left([0, T] ; H_{1}^{*} \times H_{0}^{*}\right)\right)$.

The key result in this section is the following regularity property of the "gain" $\mathcal{B}^{*} P$.

Proposition 3.1.3: (i) $\forall t, 0 \leq t<T, \mathcal{B}^{\star} P(t) \in \mathcal{L}\left(H_{1} \times H_{0}, U\right)$ with the pointwise norm estimate

$$
\left\|\mathcal{B}^{*} P(t)\right\|_{\mathcal{L}\left(H_{1}^{*} \times H_{0}^{*}, U\right)} \leq C_{T}(T-t)^{1-\theta},
$$

where $\theta$ is as given in (2.17).

(ii) For each $Y_{0} \in H_{1} \times H_{0}$ the optimal control $u^{*}\left(\cdot, s ; Y_{0}\right)$ is given in feedback form by

$$
u^{*}\left(t, s ; Y_{0}\right)=-\mathcal{B}^{*} P(t) Y^{*}\left(t, s ; Y_{0}\right), \quad 0 \leq s \leq t<T .
$$

Proof of (i): $\forall Y_{0} \in H_{1} \times H_{0}$, we have $\forall t, 0 \leq t<T$,

$$
\begin{aligned}
\left\|\mathcal{B}^{*} P(t) Y_{0}\right\|_{H_{1}^{*} \times H_{0}^{*}} & \leq \int_{t}^{T}\left\|\mathcal{B}^{*} e^{\mathcal{A}^{*}(\tau-t)} R^{*} R \Phi(\tau, t) Y_{0}\right\|_{H_{1}^{*} \times H_{0}^{*}} d \tau \\
& \leq \int_{t}^{T} \frac{C_{0}}{(\tau-t)^{\theta}}\left\|Y_{0}\right\|_{H_{1} \times H_{0}} d \tau
\end{aligned}
$$

(after using Proposition 2.2.2(ii), duality and (2.63))

$$
=\frac{C_{0}(T-t)^{1-\theta}}{1-\theta}\left\|Y_{0}\right\|_{H_{1} \times H_{0}} .
$$


(ii): Using the characterization of $u^{*}\left(\cdot, s ; Y_{0}\right)$ given by $(2.45 \mathrm{~b})$ we have

$$
\begin{aligned}
u^{*}\left(t, s ; Y_{0}\right) & =-\int_{t}^{T} \mathcal{B}^{*} e^{\mathcal{A}^{*}(\tau-t)} R^{*} R\left[e^{\mathcal{A}(\tau-s)} Y_{0}-L_{s} u^{*}\left(\cdot, s ; Y_{0}\right)(\tau)\right] d \tau \\
& =-\int_{t}^{T} \mathcal{B}^{*} e^{\mathcal{A}^{*}(\tau-t)} R^{*} R \Phi(\tau, s) Y_{0} d \tau \\
& \left.=-\mathcal{B}^{*} P(t) \Phi(\tau, t) \Phi(t, s) Y_{0} \quad \text { (after using property }(3.2 \mathrm{~b})\right) \\
& =-\mathcal{B}^{*} P(t) Y^{*}\left(t, s ; Y_{0}\right) .
\end{aligned}
$$

Proposition 3.1.4. (i) For $0 \leq t \leq T$ we have $\forall Y_{0}, Y_{1} \in H_{1} \times H_{0}$,

$$
\begin{aligned}
\left\langle P(t) Y_{0}, Y_{1}\right\rangle= & \int_{t}^{T}\left(R \Phi(\tau, t) Y_{0}, R \Phi(\tau, t) Y_{1}\right)_{Z} \\
& +\int_{t}^{T}\left(\mathcal{B}^{*} P(\tau) \Phi(\tau, t) Y_{0}, \mathcal{B}^{*} P(\tau) \Phi(\tau, t)\right)_{U} d \tau .
\end{aligned}
$$

(ii) $P(t) \in \mathcal{L}\left(H_{1} \times H_{0}, H_{1}^{*} \times H_{0}^{*}\right)$ is self-adjoint and positive semidefinite.

(iii) With $J_{t}$ as defined in (2.38), we have that the constrained minimum of $J_{t}$ corresponding to the minimizer $\left[Y^{*}\left(\cdot, t ; Y_{0}\right), u^{*}\left(\cdot, t ; Y_{0}\right)\right]^{T}$ is

$$
J_{t}\left(Y^{*}\left(\cdot, t ; Y_{0}\right), u^{*}\left(\cdot, t ; Y_{0}\right)\right)=\left\langle P(t) Y_{0}, Y_{0}\right\rangle,
$$

where the duality product is taken between $H_{1} \times H_{0}$ and $H_{1}^{*} \times H_{0}^{*}$.

Proof of (i): Using the definition of $P(t)$ in (3.3) we have for $Y_{0}, Y_{1} \in H_{1} \times H_{0}$,

$$
\left\langle P(t) Y_{0}, Y_{1}\right\rangle=\int_{t}^{T}\left(R \Phi(\tau, t) Y_{0}, R e^{\mathcal{A}(\tau-t)} Y_{1}\right)_{Z} d \tau
$$

Now using (3.1) and (2.36), we have that $\forall Y \in H_{1} \times H_{0}$,

$$
R \Phi(\tau, t) Y=R e^{\mathcal{A}(\tau-t)} Y+R L_{t} u^{*}(\cdot, t ; Y)(\tau) ;
$$

applying (3.11) to (3.10) then yields

$$
\begin{aligned}
\left\langle P(t) Y_{0}, Y_{1}\right\rangle= & \int_{t}^{T}\left(R \Phi(\tau, t) Y_{0}, R \Phi(\tau, t) Y_{1}\right)_{Z} d \tau \\
& -\int_{t}^{T}\left(R \Phi(\tau, t) Y_{0}, R L_{t} u^{*}\left(\cdot, t ; Y_{1}\right)(\tau)\right)_{Z} d \tau \\
= & \int_{t}^{T}\left[\left(R \Phi(\tau, t) Y_{0}, R \Phi(\tau, t) Y_{1}\right)+\left(u^{*}\left(\tau, t ; Y_{0}\right), u^{*}\left(\tau, t ; Y_{1}\right)\right)\right] d \tau,
\end{aligned}
$$

after taking the adjoints of $R$ and $L_{t}$ and using the characterization of $u^{*}\left(\cdot, t ; Y_{0}\right)$ in $(2.45 \mathrm{~b})$; the result follows upon applying Proposition 3.1.3(ii). (ii) and (iii) follow immediately from (i). 
3.2. Derivation of the Riccati Equation. In this section, we show that $P(t)$ defined by (3.3) satisfies the Differential Riccati Equation (DRE). we first prove a property of $\Phi(\cdot, \cdot)$ regarding differentiation in the second variable. This result is a key element in the derivation of the DRE.

Lemma 3.2.1. Let $\Phi(\cdot, \cdot)$ be as defined in (3.1); then $\forall \tau, 0 \leq \tau<T$ and $Y_{0} \in D(\mathcal{A})$, we have that $\frac{d}{d \tau} \Phi(\cdot, \tau) Y_{0}$ exists as an element of $C_{\theta}\left([\tau, T] ; H_{1} \times H_{0}\right)$ (where $\theta$ is as given in (2.17), and moreover,

$$
\frac{d}{d \tau} \Phi(\cdot, \tau) Y_{0}=\left(I_{\tau}+L_{\tau} L_{\tau}^{*} R^{*} R\right)^{-1}\left[e^{\mathcal{A}(\cdot-\tau)} \mathcal{B} L_{\tau}^{*} R^{*} R \Phi(\cdot, \tau)(\tau)-e^{\mathcal{A}(\cdot-\tau)} \mathcal{A} Y_{0}\right]
$$

Proof: Using the definition of $Y^{*}\left(\cdot, \tau ; Y_{0}\right)$ in $(2.39 \mathrm{~b})$ and the representation of $u^{*}\left(\cdot, \tau ; Y_{0}\right)$ in $(2.45 \mathrm{~b})$, we have that for $Y_{0} \in H_{1} \times H_{0}$

$$
\Phi(\cdot, \tau) Y_{0}=\left(I_{\tau}+L_{\tau} L_{\tau}^{*} R^{*} R\right)^{-1} e^{\mathcal{A}(\cdot-\tau)} Y_{0},
$$

after using the fact that $I_{\tau}+L_{\tau} L_{\tau}^{*} R^{*} R$ is invertible on $L^{2}\left(\tau, T ; H_{1} \times H_{0}\right)$ (by Lax-Milgram). Thus for fixed $t, \tau<t \leq T$, and $Y_{0} \in D(\mathcal{A})$,

$$
\begin{array}{r}
\frac{d}{d \tau} \Phi(t, \tau)+\frac{d}{d \tau}\left(L_{\tau} L_{\tau}^{*} R^{*} R \Phi(\cdot, \tau) Y_{0}(t)\right) \\
=-e^{\mathcal{A}(t-\tau)} \mathcal{A} Y_{0} \text { in } H_{1} \times H_{0},
\end{array}
$$

where each term on the LHS of (3.15) is well defined initially (in the variable $\tau$ ) at least in the sense of distributions, i.e., as elements of $\mathcal{D}^{\prime}\left(0, t ; H_{1} \times H_{0}\right)$ (see [16, p. 101]). Now for fixed $t$, the distributional derivative of $L_{\tau} L_{\tau}^{*} R^{*} R \Phi(\cdot, \tau)(t)$ is

$$
\begin{array}{r}
\frac{d}{d \tau}\left(L_{\tau} L_{\tau}^{*} R^{*} R \Phi(\cdot, \tau) Y_{0}(t)\right)=-e^{\mathcal{A}(t-\tau)} \mathcal{B}\left(L_{\tau}^{*} R^{*} R \Phi(\cdot, \tau) Y_{0}\right)(\tau) \\
+\left(L_{\tau} L_{\tau}^{*} R^{*} R \frac{d}{d \tau} \Phi(\cdot, \tau) Y_{0}\right)(t) \text { in } \mathcal{D}^{\prime}\left(0, t ; H_{1} \times H_{0}\right) ;
\end{array}
$$

thus using (3.15) and (3.16) we have for fixed $t, \tau<t \leq T$,

$$
\begin{aligned}
& {\left[\left(I_{\tau}+L_{\tau} L_{\tau}^{*} R^{*} R\right) \frac{d}{d \tau} \Phi(\cdot, \tau) Y_{0}\right](t)} \\
& \quad=e^{\mathcal{A}(t-\tau)} \mathcal{B}\left(L_{\tau}^{*} R^{*} R \Phi(\cdot, \tau) Y_{0}\right)(\tau)-e^{\mathcal{A}(t-\tau)} \mathcal{A} Y_{0} \text { in } \mathcal{D}^{\prime}\left(0, t ; H_{1} \times H_{0}\right)
\end{aligned}
$$

(given that $Y_{0} \in D(\mathcal{A})$ ). But for fixed $\tau$ the function

$$
e^{\mathcal{A}(\cdot-\tau)} \mathcal{B}\left[\left(L_{\tau}^{*} R^{*} R \Phi(\cdot, \tau) Y_{0}\right)(\tau)\right]-e^{\mathcal{A}(\cdot-\tau)} \mathcal{A} Y_{0}
$$

is an element of $C_{\theta}\left([\tau, T] ; H_{1} \times H_{0}\right)$, and we can use Propositions 2.4 .2 and 2.4 .3 and the same boot-strap argument employed in the proof of Lemma 2.4.1 to find that $I_{\tau}+L_{\tau} L_{\tau}^{*} R^{*} R$ is boundedly invertible on $C_{\theta}\left([\tau, T] ; H_{1} \times H_{0}\right)$. We can thus deduce that $\frac{d}{d \tau} \Phi(\cdot, \tau) Y_{0}$ can be taken as an element of $C_{\theta}\left([\tau, T] ; H_{1} \times H_{0}\right)$ and representation (3.13) is valid. 
Lemma 3.2.2. (i) $P(t)$ as defined in (3.9) satisfies the following Differential Riccati Equation (DRE): $\forall Y_{0}, Y_{1} \in D(\mathcal{A})$ and $\forall t \in(0, T)$,

$$
\begin{aligned}
\left\langle\dot{P}(t) Y_{0}, Y_{1}\right\rangle= & -\left\langle R^{*} R Y_{0}, Y_{1}\right\rangle-\left\langle P(t) \mathcal{A} Y_{0}, Y\right\rangle \\
& -\left\langle P(t) Y_{0}, \mathcal{A} Y_{1}\right\rangle-\left\langle\mathcal{B}^{*} P(t) Y_{0}, \mathcal{B}^{\star} P(t) Y_{1}\right\rangle,
\end{aligned}
$$

where the duality pairing $\langle\cdot, \cdot\rangle$ is taken between $H_{1} \times H_{0}$ and $H_{1}^{*} \times H_{0}^{*}$.

(ii) The solution $P(t)$ is unique within the class of self-adjoint operators $\hat{P}(t) \in \mathcal{L}\left(H_{1} \times H_{0}\right)$ which satisfy $\forall Y \in H_{1} \times H_{0}$

$$
\mathcal{B}^{*} \hat{P}(t) Y \in L^{\infty}(0, T ; U),
$$

$\forall 0<t<T$.

Proof of (i): $\forall Y_{0}, Y_{1} \in D(\mathcal{A})$ and $\forall t$, we have by the definition of $P(t)$,

$$
\left\langle P(t) Y_{0}, Y_{1}\right\rangle=\int_{t}^{T}\left\langle R^{*} R \Phi(\tau, t) Y_{0}, e^{\mathcal{A}(\tau-t)} Y_{1}\right\rangle d \tau
$$

and differentiating both sides yields

$$
\begin{aligned}
& \frac{d}{d t}\left\langle P(t) Y_{0}, Y_{1}\right\rangle \\
& \quad=-\left\langle R^{*} R Y_{0}, Y_{1}\right\rangle+\int_{t}^{T} \frac{d}{d t}\left\langle R^{*} R \Phi(\tau, t) Y_{0}, e^{\mathcal{A}(\tau-t)} Y_{1}\right\rangle d \tau
\end{aligned}
$$

Now using Lemma 3.2.1 to move the differentiation above inside the bracket yields

on the RHS of (3.21)

$$
\begin{aligned}
& =-\left\langle R^{*} R Y_{0}, Y_{1}\right\rangle \\
& -\int_{t}^{T}\left\langle R^{*} R\left[\left(I_{t}+L_{t} L_{t}^{*} R^{*} R\right)^{-1} e^{\mathcal{A}(\cdot-t)} \mathcal{A} Y_{0}\right](\tau), e^{\mathcal{A}(\tau-t)} Y_{1}\right\rangle d \tau \\
& +\int_{t}^{T}\left\langle R^{*} R\left[\left(I_{t}+L_{t} L_{t}^{*} R^{*} R\right)^{-1} e^{\mathcal{A}(\cdot-t)} \mathcal{B}\left(L_{t}^{*} R^{*} R \Phi(\cdot, t) Y_{0}\right)(t)\right](\tau), e^{\mathcal{A}(\tau-t)} Y_{1}\right\rangle d \tau \\
& -\int_{t}^{T}\left\langle R^{*} R \Phi(\tau, t) Y_{0}, e^{\mathcal{A}(\tau-t)} \mathcal{A} Y_{1}\right\rangle d \tau \\
& =-\left\langle R^{*} R Y_{0}, Y_{1}\right\rangle-\left\langle P(t) \mathcal{A} Y_{0}, Y_{1}\right\rangle \\
& +\left\langle B^{*} P(t) Y_{0}, \int_{t}^{T} B^{*} e^{\mathcal{A}^{*}(\tau-t)}\left[\left(I_{t}+L_{t} L_{t}^{*} R^{*} R\right)^{-*} R^{*} R e^{\mathcal{A}(\cdot-t)} Y_{1}\right](\tau) d \tau\right\rangle \\
& -\left\langle P(t) Y_{0}, \mathcal{A} Y_{1}\right\rangle .
\end{aligned}
$$

Now from Proposition 3.1.4(ii), $P(t)=P^{*}(t)$ and from (3.14),

$$
\Phi(\cdot, t) Y_{0}=\left(I_{t}+L_{t} L_{t}^{*} R^{*} R\right)^{-1} e^{\mathcal{A}(\cdot-t)} Y_{0} ;
$$


so by directly computing the adjoint $P^{*}(t)$ we have that $(3.20)$ indeed becomes

$$
-\left\langle R^{*} R Y_{0}, Y_{1}\right\rangle-\left\langle P(t) \mathcal{A} Y_{0}, Y_{1}\right\rangle+\left\langle\mathcal{B}^{*} P(t) Y_{0}, \mathcal{B}^{*} P(t) Y_{1}\right\rangle-\left\langle P(t) Y_{0}, \mathcal{A} Y_{1}\right\rangle .
$$

(ii): It suffices to prove the uniqueness of the solution (in the given class (3.19)) to the following (Riccati) Integral Equation:

$$
\begin{aligned}
\left\langle P(t) Y_{0}, Y_{1}\right\rangle & =\int_{\tau}^{T}\left(\operatorname{Re}^{\mathcal{A}(\tau-t)} Y_{0}, \operatorname{Re}^{\mathcal{A}(\tau-t)} Y_{1}\right)_{Z} d t \\
& -\int_{t}^{T}\left(\mathcal{B}^{*} P(\tau) e^{\mathcal{A}(\tau-t)} Y_{0}, \mathcal{B}^{*} P(\tau) e^{\mathcal{A}(\tau-t)} Y_{1}\right)_{U} d \tau
\end{aligned}
$$

$\forall Y_{0}, Y_{1} \in H_{1} \times H_{0}$. To this end, if $P_{1}(\cdot)$ and $P_{2}(\cdot)$ both solve the DRE for $0<t<T$ and are of the class $(3.19)$ ), then setting $Q(\cdot) \equiv P_{1}(\cdot)-P_{2}(\cdot)$, one has necessarily $\forall Y_{0}, Y_{1} \in H_{1} \times H_{0}$ that

$$
\begin{aligned}
\left\langle Q(t) Y_{0}, Y_{1}\right\rangle & =\int_{t}^{T}\left(\mathcal{B}^{*} P_{2}(\tau) e^{\mathcal{A}(\tau-t)} Y_{0}, \mathcal{B}^{*} Q(\tau) e^{c a l . A(\tau-t)} Y_{1}\right)_{U} d \tau \\
& -\int_{t}^{T}\left(\mathcal{B}^{*} Q(\tau) e^{\mathcal{A}(\tau-t)} Y_{0}, \mathcal{B}^{*} P_{1}(\tau) e^{\mathcal{A}(\tau-t)} Y_{1}\right)_{U} d \tau
\end{aligned}
$$

or

$$
Q(t) Y_{0}=\int_{t}^{T} e^{\mathcal{A}^{*}(\tau-t)}\left[\left(\mathcal{B}^{*} Q(\tau)\right)^{*} \mathcal{B}^{*} P_{2}(\tau) e^{\mathcal{A}(\tau-t)}-\left(\mathcal{B}^{*} P_{1}(\tau)\right)^{*} \mathcal{B}^{*} Q(\tau) e^{\mathcal{A}(\tau-t)}\right] Y_{0} d \tau
$$

or setting $V(t)=\mathcal{B}^{\star} Q(t)$, we have then, after applying $\mathcal{B}^{*}$ to both sides of (3.26), the equation

$$
V(t) Y_{0}=\int_{t}^{T} \mathcal{B}^{*} e^{\mathcal{A}^{*}(\tau-t)}\left[V^{*}(\tau) \mathcal{B}^{*} P_{2}(\tau) e^{\mathcal{A}(\tau-t)}-\left(\mathcal{B}^{*} P_{1}(\tau)\right)^{*} V(\tau) e^{\mathcal{A}(\tau-t)}\right] Y_{0} d \tau
$$

As $P_{1}(t), P_{2}(t)$ are in the specified class (3.19), then by Banach-Steinhaus one has the estimate $\forall Y \in H_{1} \times H_{0}, 0 \leq t \leq \tau \leq T$,

$$
\left\|\mathcal{B}^{*} P_{i}(\tau) Y\right\|_{U} \leq C_{T}\|Y\|_{H_{1} \times H_{0}}
$$

$(i=1,2)$.

Using the above estimate and taking the norm of both sides of equation (3.27) will yield

$$
\begin{aligned}
\left\|V(t) Y_{0}\right\| \leq & \int_{t}^{T} \frac{C_{T}}{(\tau-t)^{\theta}}\|V(\tau)\|\left\|Y_{0}\right\|_{H_{1} \times H_{0}} \\
& \quad \text { after using the norm estimate }(2.17)) \\
\leq & \int_{t}^{T} \frac{C_{T}}{(\tau-t)^{\theta}}\left\{\sup _{t \leq \tau \leq T}\|V(\tau)\|\right\} \\
\leq & C_{T}(T-t)^{1-\theta}\left\{\sup _{t \leq \tau \leq T}\|V(\tau)\|\right\} .
\end{aligned}
$$


Thus for $t_{0} \leq t \leq T$ we obtain

$$
\sup _{t_{0} \leq t \leq T}\|V(\tau)\| \leq C_{T}\left(T-t_{0}\right)^{1-\theta}\left\{\sup _{t \leq \tau \leq T}\|V(\tau)\|\right\},
$$

and for $T-t_{0}$ small enough, we will have that $C_{T}\left(T-t_{0}\right)^{1-\theta}$ is less than one, and uniqueness can be deduced within the class of all self-adjoint $\hat{P}(t) \in \mathcal{L}\left(H_{1} \times H_{0}\right)$ such that

$$
\mathcal{B}^{*} \hat{P}(t) Y \in L^{\infty}\left(t_{0}, T ; U\right),
$$

$\forall Y \in H_{1} \times H_{0}$ and $t \in\left(t_{0}, T\right)$. Iterating this argument and establishing uniqueness within each specified class will yield uniqueness for the entire interval after a finite number of steps.

\section{REFERENCES}

[1] G. Avalos, Sharp regularity estimates for the wave equation and its traces with prescribed boundary data, submitted to Appl. Math. and Optim.

[2] H. T. Banks, W. Fang, R. J. Silcox, and R. C. Smith, Approximation Methods for Control of Acoustic/Structure Models with Piezoceramic Actuators, NASA Contract Report 189578.

[3] H. T. Banks and R. C. Smith, Models for control in smart material structures, Proceedings for the Conference on Identification and Control of Systems Governed by Partial Differential Equations, SIAM (1993), $26-44$.

[4] A. Bensoussan, G. Da Prato, M. C. Delfour, and S. K. Mitter, Representation and Control of Infinite Dimensional Systems, Vol. II, Birkhäuser, Boston-Basel-Berlin, 1993.

[5] J. Cea, Lectures on Optimization-Theory and Algorithms, Springer-Verlag, Berlin, 1978.

[6] S. Chen and R. Triggiani, Proof of extensions of two conjectures on structural damping for elastic systems, Pacific J. Math. 136, no. 1 (1989), 15-55.

[7] S. Chen and R. Triggiani, Characterization of domains of fractional powers of certain operators arising in elastic systems and applications, J. Diff. Eqns. Vol. 88 (1990), 279-293.

[8] G. Da Prato, I. Lasiecka, R. Triggiani, A direct study of the Riccati equation arising in hyperbolic control problems, J. Diff. Eqns., Vol. 64 (1986) 26-42.

[9] F. Flandoli, Algebraic Riccati equation arising in boundary control problems, SIAM J. of Control and Optimiz., 25 (1987), 612-636.

[10] F. Flandoli, I. Lasiecka, and R. Triggiani, Algebraic Riccati equations with nonsmoothing observation arising in hyperbolic and Euler-Bernoulli equations, Ann. Matem. Pura e Appli., Vol. CLiii (1988), 307382 .

[11] P. Grisvard, Caracterization de qualques espaces d'interpolation, Arch. Rational Mechanics and Analysis 25 (1967), 40-63.

[12] I. Lasiecka, Unified theory for abstract parabolic boundary problems: A semigroup approach, Appl. Math. Optim. 6 (1980), pp. 287-233.

[13] I. Lasiecka and R. Triggiani, Differential and Algebraic Riccati Equations with Applications to Boundary/Point Control Problems: Continuous Theory and Approximation Theory, LNCIS, Vol. 164, SpringerVerlag, 1991.

[14] I. Lasiecka and R. Triggiani, Riccati differential equations with unbounded coefficients and nonsmooth terminal condition-the case of analytic semigroups, SIAM J. Math. Analy., Vol. 23, No. 2 (1992), pp. $449-481$.

[15] I. Lasiecka and R. Triggiani, Sharp regularity results for mixed second-order hyperbolic equations, the $L_{2}$ boundary case: Annali di Matem., Pura e Appl., IV CLVII (1990), 285-367.

[16] J. L. Lions, Optimal Control of Systems Governed by Partial Differential Equations, Springer-Verlag, 1971.

[17] J. L. Lions and E. Magenes, Non-Homogeneous Boundary Value Problems and Applications, SpringerVerlag, 1972.

[18] D. G. Luenberger, Optimization by Vector Space Methods, John Wiley, New York, 1969. 
[19] Myatake, Mixed problems for hyperbolic equations of second order, J. Math. Kyoto Univ., Vol. 130-3 (1973), 435-487.

[20] A. Pazy, Semigroups of Linear Operators and Applications to Partial Differential Equations, SpringerVerlag, New York, 1983.

[21] A. Pritchard and D. Salomon, The linear quadratic control problem for infinite dimensional systems with unbounded input and output operators, SIAM J. of Control and Optimiz., 25 (1987), 121-144.

[22] H. Triebel, Interpolation Theory, Function Spaces, Differential Operators, North-Holland, Amsterdam, 1978.

[23] I. Lasiecka, Existence and uniqueness of the solutions to second order abstract equations with nonlinear and nonmonotone boundary conditions, Nonlinear Analysis, Theory, Methods \&\& Applications, Vol. 23 No.6 (1994), 797-823.

[24] H. T. Banks and R. C. Smith, Well-posedness of a model for structural acoustic coupling in a cavity enclosed by a thin cylindrical shell, to appear in J. Math. Anal. Appl. 